Review

\title{
A microRNA Link to Glioblastoma Heterogeneity
}

\section{Sanjay K. Singh, Alenoush Vartanian, Kelly Burrell and Gelareh Zadeh*}

The Arthur and Sonia Labatt Brain Tumor Research Centre, Hospital for Sick Children, University of Toronto, Toronto, Ontario M5G 1L7, Canada; E-Mails: sanjay.singh@sickkids.ca (S.K.S.); a.albertvartanian@mail.utoronto.ca (A.V.); kelly.burrell@sickkids.ca (K.B.)

* Author to whom correspondence should be addressed; E-Mail: gelareh.zadeh@uhn.ca.

Received: 24 May 2012; in revised form: 28 July 2012 / Accepted: 21 August 2012 /

Published: 3 September 2012

\begin{abstract}
Glioblastomas (GBM) are one of the most malignant adult primary brain tumors. Through decades of research using various model systems and GBM patients, we have gained considerable insights into the mechanisms regulating GBM pathogenesis, but have mostly failed to significantly improve clinical outcome. For the most part GBM heterogeneity is responsible for this lack of progress. Here, we have discussed sources of cellular and microenvironmental heterogeneity in GBMs and their potential regulation through microRNA mediated mechanisms. We have focused on the role of individual microRNAs (miRNA) through their specific targets and miRNA mediated RNA-RNA interaction networks with the potential to influence various aspects of GBM heterogeneity including tumor neo-vascularization. We believe a better understanding of such mechanisms for regulation of GBM pathogenesis will be instrumental for future therapeutic options.
\end{abstract}

Keywords: glioma; glioblastoma; microRNA; angiogenesis; glioma stem cells; metabolism; Warburg effect

\section{Introduction}

Tumors are increasingly being viewed as complex tissue, which comprises of a heterogeneous mix of distinct cell types including cancer cells and recruited normal cells [1]. The current emphasis of tumor biology research is focused on understanding the interplay of cancer cells and tumor associated stroma and how these interactions contribute to tumorigenesis. In this review we have focused on the most common and malignant adult primary brain tumor, glioblastoma (GBM). In spite of our greater 
understanding of the biology of GBMs, GBM patients have seen only moderate improvements in outcome, as their median survival with best available treatment remains at 12-16 months [2]. It is widely accepted that the future treatment options for GBMs will greatly benefit from our improved understanding of the complex interactions between cancer cells and components of tumor microenvironment [3-13]. GBMs are characterized by significant intra-tumor regional heterogeneity comprised of areas of necrosis surrounded by a pseudopalisading proliferative edge along with a highly vascular stroma. These histological diverse regions differ in factors including the composition of subpopulation of cells, such that cells from the inner core of the tumor with higher hypoxic gradient have been shown to harbor higher concentration of glioma stem cells (GSCs) and show enhanced temozolomide (TMZ) resistance [14]. Furthermore, central and peripheral regions differ in their apoptotic index, hypoxia, migratory potential, vascularity and the therapeutic resistance, increasing the level of complexity to the microenvironmental/metabolic heterogeneity in GBMs. Here we have focused on cellular heterogeneity, their interactions in tumor microenvironment and their physiological or metabolic status as components of GBM heterogeneity. Specifically we have discussed function of individual microRNAs (miRNA) through their specific targets and miRNA mediated RNA-RNA interaction networks with potential to influence various aspects of GBM heterogeneity including tumor neo-vascularization.

We have discussed the role of miRNAs in multiple aspects of GBM biology and their clinical and functional significance in regulating factors such as angiogenesis, metabolic reprogramming and stem cell behavior. MiRNAs can function by modulating vascular integrity and angiogenesis, which is a known hallmark of high-grade gliomas, also they can alter the metabolic phenotype of GBMs by regulating key metabolic enzymes, tumor hypoxia, lactate production or signaling pathways that regulate aerobic glycolysis. In addition, miRNA have been shown to have a functional relevance in regulation of critical genes and parameters implicated in GSC behavior and differentiation.

Tumor heterogeneity arises in a multitude of ways, either as a consequence of an individual or combination of factors. For instance factors contributing to tumor heterogeneity includes: clonal evolution of cancer cells that have undergone genetic and/or epigenetic changes $[15,16]$, functional and phenotypic changes in cancer cells under the influence of different microenvironmental conditions such as hypoxia [17], and finally, cancer stem cells which while maintaining their own population can give rise to non-tumorogenic differentiated cancer cells [18-21]. GBMs have been shown to harbor glioma stem cells (GSCs) with tumorigenic potential, non-tumorigenic cancer cells, and other recruited cells; together they form heterogeneous microenvironmental niches where chemo- and radio-resistant GSCs have been shown to reside [4,13,22-24].

\section{Glioma Stem Cells (GSCs)}

Although there are no universal marker(s) for identification/enrichment of GSCs, there is growing consensus that there is a sub-population of cells in GBMs, which are selectively tumorigenic in orthotopic tumor models and share properties of normal stem/progenitor cells. Functional features of GSCs include the ability to propagate histopathologically similar tumors in orthotopic models, ability to self-renew through symmetric and asymmetric cell division and retain indefinite proliferation capacity. Apart from these essential functional criteria, GSCs have also been commonly shown to be 
both chemo- and radiation resistant [4,5,11], selectively populated in specific niches (e.g., perivascular niche (PVN)) within the tumor [6,25,26], immunosuppressive [27] and angiogenic [12]. In accordance with hierarchical cancer stem cell model, the GSCs population within a tumor may have varying degree of stemness ranging from quiescent (high label retaining [28], equivalent to adult neural stem cells), proliferating progenitors (transient amplifying population) as well as differentiated cancer cells. Thus, various aspects of GBM pathogenesis can be orchestrated by GSCs function, as they may be responsible for the establishment of cellular heterogeneity, through paracrine and autocrine signaling within the PVN to increase neo-vascularization, facilitate recruitment of cells of various origins, and maintain self-renewal. It is also notable that the PVN harbors chemo- and radio-resistant GSCs or tumor cells. GSCs have also been shown to produce pro-angiogenic factors such as vascular endothelial growth factor (VEGF) and express its cognate receptor VEGFR, thus forming an autocrine signaling loop. Blocking VEGFR in GSCs has been shown to decrease GSC proliferation and sensitize them to cell death in response to radiotherapy [29]. GSCs share some properties with neural precursor cells (NPCs). However, functionally when NPCs are present in tumor microenvironment, they harbor anti-tumor properties [30-34]. In GBM patients where young age has been associated with better prognosis, the role of NPCs is of particular interest, as this correlates with the abundance of NPCs in the brain [32], which decrease with age [35]. It has been demonstrated that NPC are able to migrate towards and infiltrate the primary glioma tumor site and also track along the invasive tumor cells and induce tumor cell death by releasing endogenous anti-tumor signals such as Cyclin D1 and D2 [34,36]. It has been suggested that signaling molecules generated from the tumors such as stromal cell-derived factor-1 (SDF-1) or VEGF provides the attractive force for NPS to migration away from the subventricular zone (SVZ) to the tumor mass. This process is age dependent and with increased age NPCs have reduced ability to migrate and produce anti-tumor signals. Results from various groups have demonstrated that GSCs are flexible in terms of their cross-lineage differentiation potential with a portion of endothelial cells (EC) within the tumor reportedly being derived from GSCs [37-40]. Thus, GSCs' contribution to GBM heterogeneity is of growing interest for understanding GBM pathobiology with clinical implications.

\section{Tumor Associated Vasculature}

Tumor vasculature plays a critical role in the maintenance, growth and progression of tumors, as they are largely responsible for oxygen and nutrient supply to the tumor. GBMs are one of the most highly vascularized solid human cancers [41]. The tumor-associated vasculature in GBMs is pathological, with hyper-proliferative and piling of ECs forming glomerular tufts [42,43]. ECs together with pericytes and vascular smooth muscle cells are intricately involved in this hyperproliferation of microvasculature [44]. These hyperproliferative areas within tumor may overlap with PVN, which in turn are important for maintenance of chemo- and radio-resistant brain tumor cells including GSCs $[6,25,26]$. ECs have been shown to be involved in extensive cross-talk with cells in the PVN [6]. The most potent pro-angiogenic factor, VEGF, is known to be produced by GSCs amongst other tumor cells, which in turn can regulate extent of tumor neo-vascularization through stimulation of ECs proliferation. Significance of this important paracrine stimulus is evident as anti-VEGF treatment results in decreased tumor neo-vascularization and growth of gliomas in xenograft models in 
preclinical studies and similar encouraging results have been demonstrated in recent clinical trials $[5,7,45]$. It has been shown that ECs secrete factors responsible for maintenance of "stemness" of GSCs in PVN [6]. Specifically, in platelet-derived growth factor (PDGF) sub-group of gliomas, nitric oxide (NO) producing enzyme eNOS is upregulated in vascular endothelium and this secreted NO diffuses into the PVN in turn helping to maintain Notch dependent self-renewal and tumorigenic potential of GSCs [25]. Additionally, in sonic hedgehog (shh) dependent GBMs, shh produced by tumor associated ECs have been shown to play a role in maintenance of GSCs [46-49]. ECs are also responsible for recruitment of pericytes to neovascularization sites resulting in the establishment of new basement membrane [50]. Recently, hypoxia-inducible factor (HIF1 $\alpha$ ) has been shown ro mediate the recruitment of pericytes to the PVN resulting in neovascularization in GBM has been documented [51]. Together with vascular smooth muscle cells, pericytes provide structural support to vasculature and lack of pericytes from vasculature results in leaky blood vessels [52,53].

\section{GBM Heterogeneity Orchestrated by Non-Physiological Levels of Oxygen, pH, and Metabolites}

Recently, the implications and better understanding of cancer energy metabolism has regained traction since the first observations by Otto Warburg that glucose metabolism in cancer cells is fundamentally different from that which occurs in normal tissue [54]. Warburg observed excessive "fermentation" of glucose to lactate in cancer cells, even when enough oxygen was present for normal respiration to take place. Although he mistakenly contributed the altered metabolism to irreversibly damaged mitochondria and hence "the origin of cancer cell", nevertheless his contributions were pivotal to our early understanding of cancer metabolism. Now we can appreciate that metabolic reprograming is possibly an adaptive response to meet the challenges of rapidly proliferating tumor cells [55]. The explanation for the switch to aerobic glycolysis or the Warburg effect as it is commonly referred to, is complex and as shown by Vander Heiden et al. [55] aerobic glycolysis satisfies the metabolic requirement of cancer cells by providing biosynthetic precursors, and that the high flux of substrates down the glucose pathway provides carbon source needed for macromolecular production such as lipids, nucleotides and proteins [55]. The metabolic adaptation in tumor microenvironment can be influenced by hypoxia, $\mathrm{pH}$ and/or the status of oncogenes and tumor suppressors; the specific role of these factors and their interactions in the establishment of the metabolic phenotype in tumor is still debated [56]. Altered expression of glucose transporters (GLUTs) and metabolic enzymes such as pyruvate kinase (PK), hexokinase II (HK2), lactate dehydrogenase (LDH), pyruvate dehydrogenase kinase (PDK) have been demonstrated in various cancers including GBMs. The expression of embryonic PK (M2) isoform is prevalent in primary tumor samples and cell lines [57,58]. PKM2 provides a metabolic and growth advantage by slowing glycolysis to allow shunting of carbon metabolites to key subsidiary biosynthetic pathways [58]. Likewise, HK2 is upregulated in cancer cells and plays a key role in regulating the Warburg effect in GBMs. Wolf et al. [59] showed that stable loss of HK2 inhibited aerobic glycolysis and led to an increase in normal oxidative respiration, decrease in lactate production and overall a switch to oxidative phosphorylation. Conversion of pyruvate (by product of glycolysis or glutaminolysis) to lactate is a reversible step governed by lactate dehydrogenase enzyme [60]. Enhanced LDHA expression is reported in tumors to facilitate 
maintenance of glycolysis by reducing intracellular lactate levels, and a concomitant increase in extracellular lactate and acidification which can promote invasion and dampening of immune surveillance [60,61]. It is also proposed that lactate can re-enter cells within tumor and act as a fuel for oxidative phosphorylation in oxygenated areas (reverse Warburg). PDK is another perpetrator of aerobic glycolysis in gliomas. It can inhibit pyruvate entry to TCA cycle by inactivation of pyruvate dehydrogenase (PDH) enzyme. Dichloroacetate (DCA) showed favorable results in phase II clinical trial in GBMs by inhibiting PDK and facilitating the switch to normal mitochondrial respiration [62].

Other molecular mechanisms involving non-metabolic genes such as known oncogenes and tumor suppressors have been implicated to converge and regulate different steps of metabolic pathways. These regulations orchestrate a state of high glucose uptake/flux, activate genes involved in the glycolysis (PI3K/AKT/P53 pathway), upregulate glutaminolysis (P53, MYC) or lipid synthesis (PI3K/AKT) and alleviate glycolysis repression (P53) [55]. The interplay of metabolic enzymes with transcriptional regulators and tumor metabolites may establish a heterogeneous metabolic microenvironment within different grades of gliomas. Since GBMs are known to reside in a heterogeneous metabolic microenvironment, this can impact the development, progression and survival of GBM patients. Inadequate vascular function, metabolic reprogramming, hypoxia, oxidative stress, acidity or $\mathrm{pH}$ imbalance in combination with GBMs intra-tumor regional heterogeneity (including genetic heterogeneity [63]) are some of many factor which has to be take into consideration during the prognostication, treatment, therapy stratification and therapeutic resistance.

\section{Tumor Hypoxia}

Tumor hypoxia has been recognized as one of the most prominent features of the dynamic neoplastic microenvironment. Within tumor hypoxia is dynamic [64]. In addition to the diffusion-limited hypoxia [64], the chaotic and disorganized tumor vascular network leads to fluctuating changes in pO2. Thus, solid tumors frequently harbor population of cancer cells with dynamic oxygen gradients that can drive and maintain aggressive tumor behavior, genetic instability, increased invasion, angiogenesis, and resistance to conventional therapy [64]. Indeed a hallmark feature of GBM histopathology is extensive cellular necrosis, presented as foci of micronecrosis encircled by pseudopalisading hypercellular neoplasmic cells [42,65-67]. Hypoxia is mostly present in high-grade gliomas, with GBMs dominated with moderate to severe hypoxic cells with a subset of oxic cells [4,5,30]. Evans et al. have demonstrated GBMs' dynamic oxygenation with oxygen concentrations ranging between $2.5 \%$ and $0.5 \%$ for mild hypoxia and $0.5 \%-0.1 \%$ for moderate/severe hypoxia [68]. Hypoxic heterogeneity within a tumor may explain the observed variations in cell death, radio-resistance and therapeutic response [64]. It has been postulated that differential growth of tumor and ECs result in an imbalance in supply and demand where oxygen and nutrient requirement of the tumors are not met. Low oxygen tension or hypoxia (chronic and acute hypoxia or anoxia) has been shown to have different effect on cellular processes. One of the major players in hypoxia adaptation is the activation and stabilization of HIF $1 \alpha, 2 \alpha$ and $3 \alpha$ and induction of slew of downstream genes with a broad functional consequence in various cancers [69]. HIFs has been recognized to play a central role in GBMs adaptation to harsh microenvironmental condition by regulating genes involved in aerobic glycolysis, angiogenesis, cell survival/death, $\mathrm{pH}$ regulation, invasion and metastasis and stem cell maintenance [70,71]. The most fundamental result of 
HIF activation is a shift in energy consumption and production by increasing aerobic glycolysis and angiogenesis, ultimately resulting in regulation of oxygen delivery and consumption [71,72]. Additional factors have been recognized that can lead to HIF1 $\alpha$ activation and accumulation in normoxic conditions (pseudohypoxia): include oncogenic mutations, mitochondrial ROS production and accumulation of TCA cycle enzymes [72]. The extent of hypoxia heterogeneity and resulting cellular and molecular alterations in GBMs thus has prognostic implications.

\section{Identifying Underlying Mechanisms for Diverse Events in Heterogeneous Tumors like GBMs}

In the era of systems biology, cancer is viewed as a manifestation of an interconnected network of intra- and extra-cellular events, which co-exist to establish and maintain the tumor microenvironment. At a molecular level, a network of transcription factors have been described, which regulate both normal (in normal development) and abnormal (in cancers and various diseases) cellular behavior [73-76]. Recently an extensive molecular network involving miRNAs have been described in GBM using most exhaustive expression database The Cancer Genome Atlas (TCGA) [77]. Together with experimentally validated role of miRNAs [78] and mRNA-miRNA-network [77], miRNA mediated mechanisms have potential to be that elusive layer of regulation, which may help explain the heterogeneous events important for establishment and maintenance of tumor microenvironment.

\section{7. microRNA: Biogenesis and Functions}

MicroRNAs are small (17-25 bases) non-coding RNAs which influence the expression of the majority of genes in the eukaryotic genome. This miRNA-mediated regulation of genes thus constitutes another layer of post-transcriptional gene regulation. The majority of miRNAs are transcribed in an RNA polII dependent manner [79,80], while some, such as the cluster of miRNAs on chromosome 19, have been shown to be transcribed by RNA polIII [79]. Many miRNA genes are transcribed as polycistronic transcripts, as they tend to be clustered together in a specific chromosomal location [80]. Some miRNA genes (e.g., mmu-mir-127 and mmu-mir-136) have also been reported to reside in imprinted loci in the genome and thus expressed from maternally inherited chromosome [81]. The primary miRNA transcripts (pri-miRNAs) have conserved secondary structures (stem-loop with 5' and 3' tails), which are recognized and processed by endoribonucleases complexes in the nucleus and cytoplasm. The nuclear endoribonuclease Drosha and DGCR8 (microprocessor complex) excise the stem-loop of approximately 70 nucleotides with a 2-nucleotide (nt) 3' overhang to produce pre-miRNA [82]. However, mirtron are processed by splicing machinery and do not require microprocessor activity for their maturation steps in nucleus but follow similar maturation steps in cytoplasm as the rest of the miRNAs $[83,84]$. These pre-miRNAs and mirtrons are then exported out of nucleus by RanGTP and Exportin-5 complex in a GTP-dependent manner [85-87]. In the cytoplasm, the pre-miRNA stem loop is further processed by the Dicer. The Dicer forms a complex with pre-miRNA, the two RNaseIII domains of Dicer cleave the pre-miRNA separated by 2 nucleotides roughly about 22 nucleotides away from the termini generating two nucleotide overhang at the 3' ends [88]. Functional miRNA-ribonucleoprotein complex is known as the miRNA-RNA-Induced Silencing Complex (miRNA-RISC). The miRNA duplex produced after Dicer activity then unwinds and binds to Argonaute (Ago) protein to form the core of the functional miRNA-RISC [89]. Argonaute family member AGO2 has RNaseH-like PIWI 
(P-element-induced wimpy testis) domain and is capable of cleavage of target RNA at the center of siRNA-mRNA pair [90-92]. The interaction between miRNP complex and its target mRNA is RNA guided and involves specific base-pairing interactions between miRNA and partially complementary sequences located (miRNA response elements, MREs) in the target RNA (e.g., 3'UTR). Specifically, the extent of complementarity to miRNA positions 2 and 8 (seed sequences/region) defines the affinity of miRNA-target RNA interaction [93-97]. The mechanisms of miRNA mediated repression includes translational repression, mRNA de-adenylation and degradation, and sequestration of mRNA to sub-cellular bodies [98].

\section{8. miRNA Targets with Established Role in Gliomagenesis}

miRNAs are mostly negative regulators of gene expression. Based on their expression levels and the genes they target in a given cellular context, miRNAs can broadly be grouped as oncogenic miRNAs or tumor suppressor miRNAs. Work from various groups studying individual miRNA function or global expression profiles of miRNA in gliomas, has established that miRNAs do in fact play a crucial role in different aspects of gliomagenesis. In gliomas, miRNA mediated mechanisms regulate a range of cellular functions which include: cell viability, cell proliferation, cell migration and invasion, apoptosis, tumor growth, cell cycle, chemo- and radio resistance, angiogenesis, tumor metabolism, and maintenance of "stemness" status of GSC [99]. Table 1 shows miRNAs involved in various aspects of GBMs and their relevant targets. The oncogenic miRNAs with known function/targets in gliomas (studied in tumors and/or glioma cells) include: miR-21, miR-221/222, miR-296, miR-10b, miR-17, miR-195, miR-455-3p, miR-10a*, miR-182, miR-451, miR-17-92 cluster etc.; and miRNAs with tumor suppressor activity include: miR-181, miR15b, miR-146b, miR-125b, miR-153, let-7, miR-153, miR-184, miR-7, miR-137, miR-128, miR-34a etc. [99]. Multiple signaling pathways that are deregulated in gliomas such as P53-, TGF $\beta$-, Apoptotic-, Interferon (IFN $\alpha /$ IFN $\beta$ )-, Notch-, NF-кB-, EGFR-, and PTEN/PI3K/AKT-pathways could potentially be targeted by miRNAs [100]. Some miRNAs have more implications in GBM pathogenesis due to their wider targets, more robust miRNA binding sites, broader functional coverage and their multiple roles when compared to other miRNAs. More consideration should be given to these miRNAs, miRNA-221/222 in having a potential role in angiogenesis, invasiveness and resistance to therapy $[101,102])$ due to their broader functional relevance. Table 2 is compiled using experimentally validated miRNA-mRNA pairs and target genes were grouped according to their known function in specific pathways using DAVID [103,104]. The miRNAs with known levels in GBMs are color coded [105]. In the following sections we have attempted to highlight pro- and anti-tumorigenic function of miRNAs and how their aberrations can mechanistically modulate multiple aspects of GBMs.

Table 1. List of miRNAs discussed in the text with their relevant functions and targets in GBMs or other cancers.

\begin{tabular}{|l|l|l|}
\hline MicroRNA functions & MicroRNAs & Relevant targets \\
\hline Glioma stem cells & $\begin{array}{l}\text { miR-124 } \\
\text { miR-137 }\end{array}$ & $\begin{array}{l}\text { PTBP1, CDK6, SCP1, LAMC1, ITGB1 } \\
\text { CDK6 }\end{array}$
\end{tabular}


Table 1. Cont.

\begin{tabular}{|c|c|c|c|}
\hline MicroRNA functions & MicroRNAs & Relevant targets & Reference \\
\hline Glioma stem cells & $\begin{array}{l}\text { miR-128 } \\
\text { miR-7 } \\
\text { miR-425 } \\
\text { miR-486 } \\
\text { miR-451 } \\
\text { miR-34a } \\
\text { miR-326 }\end{array}$ & $\begin{array}{l}\text { Bmi-1, E2F3A } \\
\text { EGFR, IRS2 } \\
\text { Notch1, Notch2 } \\
\text { Notch }\end{array}$ & [106-113] \\
\hline Pro-agiomiRs & $\begin{array}{l}\text { miR-126, } \\
\text { miR-17-92 cluster } \\
\text { miR-378 } \\
\text { miR-296 } \\
\text { miR-21 } \\
\text { miR-210 } \\
\text { miR-130a } \\
\text { miR-125b } \\
\text { miR-101 }\end{array}$ & $\begin{array}{l}\text { Spred-1, PIK3R2 } \\
\text { TSP-1 } \\
\text { Sufu, Fus-1 } \\
\text { HGS } \\
\text { Ephrin-A3 } \\
\text { HOXA5, GAX } \\
\text { MAZ } \\
\text { EZH2 }\end{array}$ & [114-126] \\
\hline Anti-angiomiRs & $\begin{array}{l}\operatorname{miR} 221 / 222 \\
\operatorname{miR}-320\end{array}$ & $\begin{array}{l}\text { c-Kit } \\
\text { IGF-1 }\end{array}$ & {$[127,128]$} \\
\hline $\begin{array}{l}\text { Aerobic glycolysis \& } \\
\text { related signaling } \\
\text { pathways }\end{array}$ & $\begin{array}{l}\text { miR-326 } \\
\text { miR-21 } \\
\text { miR-26a, } \\
\text { miR-221/222 } \\
\text { miR-451 } \\
\text { miR-128 } \\
\text { miR-25 } \\
\text { mir-32 } \\
\text { miR-34a } \\
\text { miR-155/143 } \\
\text { mir-23a/b }\end{array}$ & $\begin{array}{l}\text { PKM2 } \\
\text { PI3K/AKT/P53 } \\
\text { PI3K/AKT } \\
\text { PI3K/AKT } \\
\text { LKB1/AMPK } \\
\text { AKT } \\
\text { P53 } \\
\text { P53 } \\
\text { MYC } \\
\text { CEBPb } \\
\text { MYC }\end{array}$ & [129-131] \\
\hline Hypoxamirs & $\begin{array}{l}\operatorname{miR}-20 b \\
\text { miR-210 } \\
\text { miR-199a }\end{array}$ & $\begin{array}{l}\text { Sirt1 } \\
\text { ISCU1/2 } \\
\text { HIF-1 } \alpha, \text { Sirt1 }\end{array}$ & [132-135] \\
\hline
\end{tabular}

Table 2. Experimentally validated targets of miRNAs are components of pathways known to play key role in GBM biology. MicroRNAs shown underlined are over expressed and shown in bold are under expressed miRNAs in GBMs.

\begin{tabular}{|c|c|l|}
\hline S. No. & Gene name & miRNA(s) \\
\hline 1 & PDGFA & hsa-let-7d \\
\hline 2 & PDGFB & hsa-miR-146b-3p \\
\hline 3 & EGFR & $\begin{array}{l}\text { hsa-miR-1, hsa-miR-128, hsa-miR-146a, hsa-miR-16, hsa-miR-21, } \\
\text { hsamiR-7 }\end{array}$ \\
\hline
\end{tabular}


Table 2. Cont.

\begin{tabular}{|c|c|c|}
\hline \multicolumn{3}{|r|}{ Glioma de novo pathway (KEGG) } \\
\hline S. No. & Gene name & miRNA(s) \\
\hline 4 & PDGFRA & hsa-let-7b \\
\hline 5 & PDGFRB & hsa-miR-224 \\
\hline 6 & IGF1R & $\begin{array}{l}\text { hsa-miR-7, hsa-miR-122, hsa-miR-133b, hsa-miR-138, hsa-miR- } \\
\text { 145, hsamiR-194 }\end{array}$ \\
\hline 7 & GRB2 & hsa-miR-433 \\
\hline 8 & $\begin{array}{l}\text { CALM2, } \\
\text { CALM3 }\end{array}$ & hsa-miR-1 \\
\hline 9 & CAMK2G & hsa-miR-219-5p \\
\hline 10 & HRAS & hsa-let-7a, hsa-miR-143, hsa-miR-181a, \\
\hline 11 & KRAS & $\begin{array}{l}\text { hsa-let-7a, hsa-let-7g, hsa-miR-143, hsa-miR-155, hsa-miR-181c, } \\
\text { hsa-miR-18a*, hsa-miR-96 }\end{array}$ \\
\hline 12 & NRAS & hsa-let-7a, hsa-let-7b, hsa-let-7c, hsa-miR-20a \\
\hline 13 & PIK3R1 & hsa-miR-29a \\
\hline 14 & PIK3R2 & hsa-miR-126 \\
\hline 15 & PTEN & $\begin{array}{l}\text { hsa-miR-106b, hsa-miR-141, hsa-miR-17, hsa-miR-18a, hsa-miR- } \\
\text { 19a, hsa-miR-19b, hsa-miR-20a, hsa-miR-21, hsa-miR-214, hsa- } \\
\text { miR-216a, hsa-miR-217, hsa-miR-221, hsa-miR-222, hsa-miR-26a, } \\
\text { hsa-miR-494 }\end{array}$ \\
\hline 16 & $A R A F$ & hsa-miR-124 \\
\hline 17 & RAF1 & hsa-miR-125b, hsa-miR-7 \\
\hline 18 & AKT1 & hsa-miR-125b, hsa-miR-149*, hsa-miR-185, hsa-miR-451 \\
\hline 19 & MAP2K1 & hsa-miR-34a, hsa-miR-424 \\
\hline 20 & MAPK1 & hsa-miR-199b-3p \\
\hline 21 & CDKN2A & 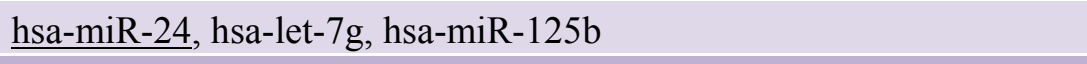 \\
\hline 22 & TP53 & $\begin{array}{l}\text { hsa-miR-25, hsa-miR-30d, hsa-miR-612, hsa-miR-125a-5p, hsa- } \\
\text { miR-125b, hsa-miR-1285, hsa-miR-15a, hsa-miR-16, hsa-miR-221, } \\
\text { hsa-miR-222 }\end{array}$ \\
\hline 23 & CDKN1A & $\begin{array}{l}\text { hsa-miR-106a, hsa-miR-106b, hsa-miR-125a-5p, hsa-miR-132, hsa- } \\
\text { miR-145, hsa-miR-146a, hsa-miR-146b-5p, hsa-miR-17, hsa-miR- } \\
\text { 182, hsa-miR-208a, hsa-miR-208b, hsa-miR-20a, hsa-miR-20b, hsa- } \\
\text { miR-28-5p, hsa-miR-298, hsa-miR-299-5p, hsa-miR-302a, hsa-miR- } \\
\text { 345, hsa-miR-363, hsa-miR-372, hsa-miR-423-3p, hsa-miR-503, hsa- } \\
\text { miR-515-3p, hsa-miR-519d, hsa-miR-519e, hsa-miR-520a-3p, hsa- } \\
\text { miR-520b, hsa-miR-520h, hsa-miR-572, hsa-miR-639, hsa-miR-654- } \\
\text { 3p, hsa-miR-657, hsa-miR-93, hsa-miR-942, hsa-miR-96 }\end{array}$ \\
\hline 24 & CDKN2A & hsa-let-7g, hsa-miR-125b, hsa-miR-24 \\
\hline 25 & CCND1 & $\begin{array}{l}\text { hsa-let-7b, hsa-miR-106b, hsa-miR-15a, hsa-miR-15b, hsa-miR-16, } \\
\text { hsa-miR-16-1*, hsa-miR-17, hsa-miR-193b, hsa-miR-19a, hsa-miR- } \\
\text { 20a, hsa-miR-302a, hsa-miR-302c, hsa-miR-34a, hsa-miR-424, hsa- } \\
\underline{\text { miR-449a, hsa-miR-503 }}\end{array}$ \\
\hline 26 & CDK4 & $\begin{array}{l}\text { hsa-miR-124, hsa-miR-145, hsa-miR-24, hsa-miR-302a, hsa-miR- } \\
\text { 34a, hsa-miR-34b, hsa-miR-34b*, hsa-miR-34c-5p }\end{array}$ \\
\hline
\end{tabular}


Table 2. Cont.

\begin{tabular}{|c|c|c|}
\hline \multicolumn{3}{|r|}{ Glioma de novo pathway (KEGG) } \\
\hline S. No. & Gene name & miRNA(s) \\
\hline 27 & CDK6 & 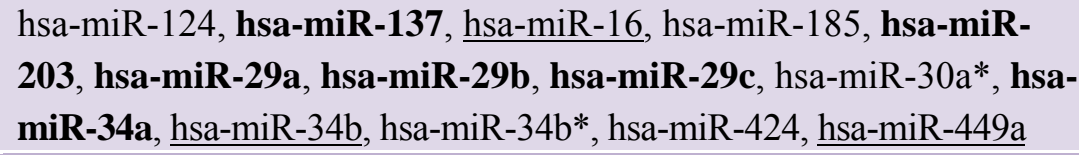 \\
\hline 28 & $R B 1$ & $\begin{array}{l}\text { hsa-miR-106a, hsa-miR-106b, hsa-miR-17, hsa-miR-20a, } \\
\text { hsa-miR-23b, hsa-miR-26a, hsa-miR-335, hsa-miR-675 }\end{array}$ \\
\hline 29 & E2F1 & $\begin{array}{l}\text { hsa-let-7a, hsa-miR-106a, hsa-miR-106b, hsa-miR-126, hsa-miR- } \\
\text { 149*, hsa-miR-17, hsa-miR-20a, hsa-miR-21, hsa-miR-223, hsa- } \\
\text { miR-23b, hsa-miR-330-3p, hsa-miR-34a, hsa-miR-93, hsa-miR-98 }\end{array}$ \\
\hline \multicolumn{3}{|r|}{ TGF beta Signaling pathway (KEGG) } \\
\hline S. No. & Gene name & miRNA(s) \\
\hline 1 & BMP7 & hsa-miR-22, hsa-miR-342-3p \\
\hline 2 & THBS1 & $\begin{array}{l}\text { hsa-let-7a, hsa-let-7b, hsa-miR-1, hsa-miR-17, hsa-miR-20a, } \\
\text { hsa-miR-30a*, hsa-miR-92a, hsa-miR-98 }\end{array}$ \\
\hline 3 & TGFB1 & $\underline{\text { hsa-miR-24 }}$ \\
\hline 4 & TGFB2 & hsa-miR-141 \\
\hline 5 & TGFB3 & hsa-miR-29a \\
\hline 6 & $\begin{array}{l}\text { LEFTY1, } \\
\text { LEFTY2 }\end{array}$ & hsa-miR-302a, hsa-miR-302d \\
\hline 7 & BMPR1B & hsa-miR-125b \\
\hline 8 & $B M P R 2$ & $\begin{array}{l}\text { hsa-miR-129-5p, hsa-miR-17, hsa-miR-19a, hsa-miR-19b, hsa-miR- } \\
\text { 20a, hsa-miR21, hsa-miR-92a }\end{array}$ \\
\hline 9 & TGFBR1 & hsa-let-7c, hsa-miR-128, hsa-miR-204 \\
\hline 10 & TGFBR2 & $\begin{array}{l}\text { hsa-miR-17, hsa-miR-18a, hsa-miR-19a, hsa-miR-19b, hsa-miR-204, } \\
\text { hsa-miR-20a, hsa-miR-21, hsa-miR-302b, hsa-miR-372, } \\
\text { hsa-miR-590-5p, hsa-miR-92a }\end{array}$ \\
\hline 11 & ACVR1 & hsa-miR-197 \\
\hline 12 & ACVR2A & $\underline{\text { hsa-miR-16 }}$ \\
\hline 13 & ACVR1C & $\begin{array}{l}\text { hsa-miR-122, hsa-miR-147, hsa-miR-22, hsa-miR-376a, } \\
\text { hsa-miR376a*, hsa-miR-376b, hsa-miR-376c, hsa-miR-412 }\end{array}$ \\
\hline 14 & SMAD1 & hsa-miR-155, hsa-miR-26a \\
\hline 15 & SMAD2 & $\underline{\text { hsa-miR-155 }}$ \\
\hline 16 & SMAD5 & $\underline{\text { hsa-miR-155 }}$ \\
\hline 17 & RHOA & hsa-miR-122, hsa-miR-155, hsa-miR-185, hsa-miR-31 \\
\hline 18 & ROCK1 & hsa-miR-146a, hsa-miR-584 \\
\hline 19 & ROCK2 & hsa-miR-138 \\
\hline 20 & SMAD4 & $\begin{array}{l}\text { hsa-miR-17, hsa-miR-18a, hsa-miR-19a, hsa-miR-20a, } \\
\text { hsa-miR-26a, hsa-miR-483-3p, hsa-miR-92a }\end{array}$ \\
\hline 21 & MAPK1 & $\underline{\text { hsa-miR-199b-3p }}$ \\
\hline 22 & ID1 & hsa-miR-100, hsa-miR-520h \\
\hline 23 & ID3 & hsa-miR-520h \\
\hline 24 & $R B L 1, R B L 2$ & hsa-miR-106b, hsa-miR-17, hsa-miR-20a \\
\hline
\end{tabular}


Table 2. Cont.

\begin{tabular}{|c|c|c|}
\hline \multicolumn{3}{|r|}{ TGF beta Signaling pathway (KEGG) } \\
\hline S. No. & Gene name & miRNA(s) \\
\hline 25 & E2F5 & hsa-let-7b, hsa-miR-192, hsa-miR-34a \\
\hline 26 & CREBBP & hsa-miR-324-3p \\
\hline 27 & EP300 & $\begin{array}{l}\text { hsa-miR-182, hsa-miR-194, hsa-miR-200b, hsa-miR-200c, } \\
\text { hsa-miR-26b, hsa-miR-374a, hsa-miR-429 }\end{array}$ \\
\hline 28 & SP1 & hsa-miR-124, hsa-miR-218, hsa-miR-29b \\
\hline 29 & MYC & $\begin{array}{l}\text { hsa-let-7a, hsa-let-7g, hsa-miR-145, hsa-miR-17, hsa-miR-20a, } \\
\text { hsa-miR-21, hsa-miR-24, hsa-miR-26a, hsa-miR-34a, hsa-miR-34b, } \\
\text { hsa-miR-34b*, hsa-miR-34c-5p, hsa-miR-378, hsa-miR-98 }\end{array}$ \\
\hline \multicolumn{3}{|r|}{ Notch signaling pathway (KEGG) } \\
\hline S. No. & Gene name & miRNA(s) \\
\hline 1 & DLL1 & hsa-miR-34a \\
\hline 2 & JAG1 & 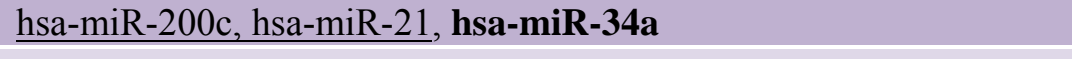 \\
\hline 3 & ADAM17 & hsa-miR-122 \\
\hline 4 & NOTCH1 & $\begin{array}{l}\text { hsa-miR-129-5p, hsa-miR-144, hsa-miR-30a, hsa-miR-326, } \\
\text { hsa-miR-34a }\end{array}$ \\
\hline 5 & NOTCH2 & hsa-miR-1, hsa-miR-16, hsa-miR-181c, hsa-miR-326, hsa-miR-34a \\
\hline 6 & NOTCH3 & hsa-miR-206 \\
\hline 7 & NOTCH4 & hsa-miR-181c \\
\hline 8 & DVL2 & hsa-miR-324-3p \\
\hline 9 & NUMB & hsa-miR-31 \\
\hline 10 & NUMBL & hsa-miR-122 \\
\hline 11 & PSEN1 & hsa-miR-562 \\
\hline 12 & CREBBP & hsa-miR-324-3p \\
\hline 13 & EP300 & $\begin{array}{l}\text { hsa-miR-182, hsa-miR-194, hsa-miR-200b, hsa-miR-200c, hsa-miR- } \\
\text { 26b, hsa-miR374a, hsa-miR-429 }\end{array}$ \\
\hline 14 & KАT2B & $\begin{array}{l}\underline{\text { hsa-miR-106b, hsa-miR-181a, hsa-miR-181b, hsa-miR-19a, }} \\
\underline{\text { hsa-miR-19b, hsa-miR-25, hsa-miR-32, hsa-miR-92a, hsa-miR-93 }}\end{array}$ \\
\hline 15 & CTBP1 & hsa-miR-137 \\
\hline 16 & HDAC1 & hsa-miR-449a \\
\hline 17 & NCOR2 & hsa-miR-10a, hsa-miR-10b \\
\hline 18 & HES1 & $\underline{\text { hsa-miR-199b-5p, hsa-miR-23a }}$ \\
\hline \multicolumn{3}{|r|}{ VEGF signaling pathway (KEGG) } \\
\hline S. No. & Gene name & miRNA(s) \\
\hline 1 & VEGFA & $\begin{array}{l}\text { hsa-miR-106a, hsa-miR-106b, hsa-miR-107, hsa-miR-125a-5p, hsa- } \\
\text { miR-126, hsa-miR-134, hsa-miR-140-5p, hsa-miR-147, hsa-miR- } \\
\text { 150, hsa-miR-15a, hsa-miR-15b, hsa-miR-16, hsa-miR-17, hsa-miR- } \\
\text { 195, hsa-miR-205, hsa-miR-20a, hsa-miR-20b, hsa-miR-29b, hsa- } \\
\text { miR-302d, hsa-miR-330-3p, hsa-miR-34a, hsa-miR-34b, hsa-miR- } \\
\text { 361-5p, hsa-miR-372, hsa-miR-373, hsa-miR-378, hsa-miR-383, } \\
\text { hsa-miR-504, hsa-miR-520g, hsa-miR-520h, hsa-miR-93 }\end{array}$ \\
\hline
\end{tabular}


Table 2. Cont.

\begin{tabular}{|c|c|c|}
\hline \multicolumn{3}{|r|}{ VEGF signaling pathway (KEGG) } \\
\hline S. No. & Gene name & miRNA(s) \\
\hline 2 & CDC42 & $\begin{array}{l}\text { hsa-miR-137, hsa-miR-185, hsa-miR-216a, hsa-miR-224, } \\
\text { hsa-miR-330-3p, hsa-miR-608 }\end{array}$ \\
\hline 3 & PIK3R1 & hsa-miR-29a \\
\hline 4 & PIK3R2 & hsa-miR-126 \\
\hline 5 & MAPK11 & hsa-miR-122 \\
\hline 6 & MAPK14 & hsa-miR-124, hsa-miR-24 \\
\hline 7 & AKT1 & hsa-miR-125b, hsa-miR-149*, hsa-miR-185, hsa-miR-451 \\
\hline 8 & HRAS & hsa-let-7a, hsa-miR-143, hsa-miR-181a \\
\hline 9 & KRAS & $\begin{array}{l}\text { hsa-let-7a, hsa-let-7g, hsa-miR-143, hsa-miR-155, hsa-miR-181c, } \\
\text { hsa-miR-18a*, hsa-miR-96 }\end{array}$ \\
\hline 10 & NRAS & hsa-let-7a, hsa-let-7b, hsa-let-7c, hsa-miR-20a \\
\hline 11 & RAF1 & hsa-miR-125b, hsa-miR-7 \\
\hline 12 & РРРЗСА & hsa-miR-145, hsa-miR-30a \\
\hline 13 & PPP3R1 & hsa-miR-30a \\
\hline 14 & NFAT5 & hsa-miR-24, hsa-miR-31 \\
\hline 15 & NFATC2 & hsa-miR-184 \\
\hline 16 & PTK2 & hsa-miR-193a-3p \\
\hline 17 & RAC1 & hsa-miR-122, hsa-miR-194 \\
\hline 18 & CASP9 & hsa-let-7a, hsa-miR-133a \\
\hline 19 & PTGS2 & hsa-let-7b, hsa-miR-101, hsa-miR-16, hsa-miR-26b \\
\hline 20 & PLA2G4B & hsa-miR-338-3p \\
\hline 21 & MAPK1 & hsa-miR-199b-3p \\
\hline \multicolumn{3}{|r|}{ PDGF Signaling Pathway (Biocarta) } \\
\hline S. No. & Gene name & miRNA(s) \\
\hline 1 & PDGFA & hsa-let-7d \\
\hline 2 & PDGFRA & hsa-let-7b, \\
\hline 3 & GRB2 & hsa-miR-433 \\
\hline 4 & JAK1 & $\underline{\text { hsa-miR-17 }}$ \\
\hline 5 & RASA1 & hsa-miR-335 \\
\hline 6 & STAT1 & hsa-miR-145 \\
\hline 7 & STAT3 & hsa-miR-125b, hsa-miR-20b \\
\hline 8 & STAT5A & hsa-miR-222 \\
\hline 9 & $S R F$ & hsa-miR-122 \\
\hline 10 & FOS & hsa-miR-101, hsa-miR-221, hsa-miR-222 \\
\hline 11 & JUN & hsa-miR-30a \\
\hline 12 & HRAS & hsa-let-7a, hsa-miR-143, hsa-miR-181a, \\
\hline 13 & RAF1 & hsa-miR-125b, hsa-miR-7 \\
\hline 14 & MAP2K1 & hsa-miR-34a, hsa-miR-424 \\
\hline 15 & МАРЗК1 & hsa-miR-192 \\
\hline
\end{tabular}




\section{9. miRNAs in Glioma Stem Cells (GSCs)}

GSCs share properties with NPCs both in terms of cellular function and molecular mechanisms that govern these cellular functions. Expression levels of many key components of self-renewal machinery (e.g., Nestin, Bmi1, Olig2, Sox2 etc.) in NPCs are also expressed at high levels in GSCs [136]. NPCs during normal development are able to fully differentiate after exiting self-renewing state, GSCs on the other hand, appear to retain the self-renewal (indefinite proliferation) property while losing the capacity to terminally differentiate. MiRNAs like miR-124, miR-137, miR-128, miR-7 etc. are weakly expressed in gliomas and thus result in relieved repression of their targets (targets of these miRNAs include: CDK6, SCP1, PTBP1, LAMC1, ITGB1, BMI1, EGFR, E2F3A, IRS2 etc.). CDK6 a known promoter of G1/S phase transition has been shown to promote cell cycle in GSCs. Expression levels of has-miR-125b has been shown to be lower in GSCs and can target CDK6 [137]. BMI1 a polycomb group of genes, using mouse models it has been shown to maintain "stemness" of NSCs largely by blocking differentiation [138,139]. EGFR signaling has been shown to promote "stemness" of GSCs by activating expression of inhibitor of differentiation 3 (ID3) through SMAD5 [140]. The bone morphogenetic protein 4 (BMP4) regulated Smad signaling cascade responsible for inhibition of tumorigenic potential of GSCs [141] orchestrate their function in part by up-regulation of miR-451 [106]. This may explain sustained proliferation/self-renewal of GSCs [107-110]. CD133 ${ }^{+v e}$ GSCs when compared to CD133 ${ }^{-v e}$ glioma cells have lower expression levels of miR-425, miR-486 and miR-451 suggesting heterogeneous expression pattern for miRNAs within tumor. Activation of the Notch pathway has been implicated in the maintenance of GSCs ("stemness", proliferation and radioresistance) [111,142-144]. Notch1 and Notch2 components of Notch pathway are targets of miR-34a, a miRNA which is down-regulated in GSCs [112]. Notch is also targeted by miR-326 in GSCs [113]. Thus miRNAs that play a crucial role in maintenance of self-renewal in normal NPCs have also been shown to promote undifferentiated status of GSCs.

\section{0. miRNAs in Tumor Associated Angiogenesis}

One of the most profound features of malignant progression of solid tumors is the induction of tumor neo-vascularization [145,146]. It is well established that regulation of miRNA expression is essential for normal development and differentiation of ECs and deregulated miRNAs can compromise vascular integrity in normal development. Multiple studies have revealed microRNA-driven regulation of angiogenesis in different tumors and this list is expanding rapidly. These miRNAs, which have been termed angiomiRs [147] may function by either promoting (pro-angiomiR) or inhibiting angiogenesis (anti-angiomiR), similar to pro- and anti-angiogenic factors that modulate different players of angiogenic pathways [147]. Some of the most studied pro-angiomiRs are miR-126, miR-17-92 cluster, miR-378, miR-296, miR-21, miR-210, miR-130a and some anti-angiomiRs such as miR-221/222 or miR-320 [114-124,127,128]. The role for miR-296 in glioma angiogenesis has been extensively studied in tumor and ECs [124]. MiR-296 was shown to be induced in glioma cells and endothelium through angiogenic growth factors and mediating angiogenesis in vitro. Furthermore, inhibition of miR-296 reduced neo-vascularization in subcutaneous tumors in vivo [124]. Recently another angiomiR, miR-125b was shown to down-regulate Myc-associated zinc finger protein (MAZ) and is 
also down regulated in GBMs. As MAZ is a known transcriptional activator of VEGF, reduction in MAZ indirectly activates VEGF and augments GBM angiogenesis [125]. Thus, modulating miR-125b may have therapeutic potential given its ability to inhibit vascularization. Similarly, miR-101 promotes glioma cell proliferation, migration, and angiogenesis in vivo and in vitro [126]. MiR-101 expression was reduced in GBM samples, resulting in increased expression of its target EZH2 and indirect induction of number of angiogenic genes [126]. Direct and indirect miRNA mediated mechanisms can regulate angiogenesis and tumor progression in GBMs.

\section{1. miRNAs in Tumor Metabolism}

Here we focus on microRNA-mediated regulation of aerobic glycolysis and/or signaling pathways that regulate glycolysis in gliomas and other cancers. Recently miR-451 was proposed as a regulator of the LKB1/AMPK signaling pathway in gliomas, which may be central to mechanisms that regulate cellular adaptation or resistance to metabolic stress during altered energy availability [129]. It has been shown that miR-326 can directly targets PKM2 isoform, which is highly overexpressed in cancer cells including GBMs, and can be utilized to regulate glioma metabolism [148]. The PI3K/AKT pathway regulates several aspects of glucose metabolism such as targeting and regulating glucose transporters or enhancing aerobic glycolysis through HK2 regulation. Several micro-RNAs have been shown to regulate PI3K/AKT pathway in gliomas such as oncogenic miR-21 and miR-26a, miR-221/222 as activators of AKT, and miR-451 and miR-128 as negative regulators of AKT pathway [149-155]. Tumor suppressor P53 has a role in dampening glycolysis and enhancing mitochondrial respiration, which is compatible with its tumor suppressor function. MiR-21 has been shown to regulate multiple important components of the P53 pathway in GBM cells. MiR-25 and miR-32 are part of a P53 mediated tumor suppressor circuitry [156,157]. When overexpressed in glioma cells they result in decreased cell growth and increased survival of xenograft models. Also, miR-34a was shown to have tumor suppressor function in P53-mutant U251 cells [158]. Recently, miR-155/143 cascade was shown to promote aerobic glycolysis indirectly by regulating HK2 expression [159]. This mechanism holds promise in GBMs since HK2 is highly expressed in GBMs as compared to normal brain or low-grade astrocytomas thus promoting aerobic glycolysis [160]. MYC regulation of glutamine and glucose metabolism is well established. MYC suppression by miR-23a/b and oncogenic function of miR-17-92 cluster has been shown in B cell lymphoma and prostate cancer to promote mitochondrial glutaminolysis, aerobic glycolysis, cell proliferation and might also be important to investigate in GBMs [130,131,161].

\section{2. miRNA in Tumor Hypoxia}

Low oxygen tension or cellular hypoxia has been shown to alter miRNA expression levels (up- or down-regulated). Conversely, miRNAs can regulate cellular HIF levels and its downstream genes resulting in plethora of effects including alterations in glucose transport, glucose metabolism, TCA cycle or angiogenesis. Amongst the HIF-dependent miRNAs known as hypoxamirs [162], miR210 has been extensively studied. MiR-210 is induced by HIF-1 $\alpha$ [132] and was recently shown to be up-regulated in GBMs in hypoxic conditions in-vitro [163]. Also miR-210 was shown to repress mitochondrial metabolism under hypoxic conditions by decreasing expression of the iron-sulfur cluster 
assembly proteins ISCU1/2 [133]. This is in line with findings of Puisségur et al. regarding miR-210 mediated mitochondrial dysfunction by direct inhibition of succinate dehydrogenase subunit $\mathrm{D}$ (SDHD) and positive regulation of HIF by miR-210 (lung cancer). These functional effects mediated by miR-210 might have implications for altered metabolism in GBMs since it can modulate mitochondrial oxygen consumption and create a pseudohypoxic environment. Hypoxamirs such as miR-20b [134] in MCF-7 breast cancer cells and miR-199a in cardiomyocytes [135] can target HIF-1 $\alpha$ to suppress its expression. Under hypoxic conditions miR-199a can directly target HIF-1 $\alpha$ for inhibition, however under normoxia miR-199a derepresses and stabilizes HIF-1 $\alpha$ by inhibiting sirtuin 1 (Sirt1), which in normal conditions can augments HIF signaling by inhibit prolyl hydroxylase 2 required for stabilization of HIF-1 $\alpha$ [135]. Elaborate interactions of hypoxamirs and HIF-mediated responses add another level of complexity to hypoxic niche network. Deciphering hypoxamir regulation in different cellular context may shed a light on hypoxia-driven induction of aerobic glycolysis, angiogenesis, invasion and metastasis or stem cell phenotype in GBMs.

\section{3. miRNA Mediated Networks in Glioma}

Classically, miRNA and mRNA interaction is defined as a unidirectional regulation mechanism, where miRNA for the most part negatively regulates expression of target mRNA. This view of miRNA-mRNA interaction is challenged by competitive endogenous RNA hypothesis (ceRNA hypothesis). According to ceRNA hypothesis, mRNAs including RNAs arising from pseudogenes [164]) can negatively regulate miRNA function [165]. Over the recent years evidences for this mechanism in various cellular context (normal and cancer) has increased. Analysis of The Cancer Genome Atlas (TCGA) database revealed an extensive miRNA mediated RNA-RNA interaction network and explained mRNA function based on ceRNA hypothesis. PTEN tumor suppressor gene in glioma is discovered to be extensively integrated in the miRNA mediated RNA-RNA interaction network [166,167]. Through these newly identified miRNA-RNA networks, genes involved multiple pathways with known function in gliomagenesis are able to cross-talk and influence each other's expression (RB, PTEN, PDGFR, VEGFA, RUNX1, and STAT3). Thus, miRNA apart from their widely reported function, are key participant in an extensive RNA-RNA interaction network and establish a new layer of epigenetic regulation. Since ceRNA mechanism rely on the relative abundance of RNAs (miRNAs and mRNAs) in the cells, specific interaction network may become dysfunctional, if concentration/expression of any of the interacting RNAs were to go to either extremes (i.e., no expression or over-expression). It is therefore conceivable that miRNAs in tumor have at least two distinct modes of action: (1) classical miRNA-mRNA regulation; (2) miRNAs in ceRNA-mediated interactions. This is very exciting and hopeful time in miRNA biology, however, the generation of miRNA mediated RNA-RNA interaction network based on physiologically relevant miRNA-mRNA interactions (rather than based bioinformatics prediction models) using biochemical assays and RNA sequencing is urgently needed. Thus, miRNA mediated mechanisms by connecting and establishing cross-talk between wide arrays of aberrant pathways and cellular functions may provide a global perspective to tackle heterogeneous and hard to manage tumors like GBM. 


\section{Conclusions}

Heterogeneous composition of GBMs with respect to resident tumor cells and parenchymal cells (EC, microglia, immune cells etc.) provides an additional layer of complexity to the pathology. The major limitations in the clinic are the multifactorial nature of the disease, its molecular and metabolic heterogeneity and the presence of functionally heterogeneous tumor cell populations. The differential expression of miRNA groups brought about by factors such as diverse cellular context, tumorigenic stages or the extent of metabolic stress pose a considerable challenge in therapy and evaluation of therapeutic effectiveness; making it less feasible to develop more personalized miRNA based treatment strategies at the present time. In this review we have discussed different interactions of tumor cells including GSCs and ECs with microenvironmental factors such as hypoxia and cellular metabolism, highlighting areas of interest for future investigations. At a cellular level, cancer cells (tumorigenic and non-tumorigenic) display a dynamic interaction within tumor (paracrine, autocrine, metabolic status, and cell-cell interactions) whereby creating a pro-tumor environment. As depicted in Figure 1, in cells within tumor including GSCs and ECs, an epigenetic program orchestrated by miRNA pool together with mRNA/RNA pool can explain multiple aspects of observed heterogeneity including metabolic and physiological heterogeneity. The interaction between various cell types at least in part is manifestation of their respective microRNA mediated epigenetic program. A newly described elaborate miRNA mediated RNA-RNA network can explain multiple sources of molecular diversity, which ultimately results in a tumor with heterogeneous features. A better understanding of this epigenetic program may provide novel methods for management of GBM patients.

Figure 1. A schematic representation of possible miRNA mediated epigenetic network in tumorigenic cancer stem cells (GSCs; shown in black font color) and tumor associated normal cells (endothelial cells; shown in blue font color) within the tumor microenvironment. Double-headed black arrow represents cell-to-cell communication in tumor microenvironment and influence of extra-cellular factors on cellular functions.

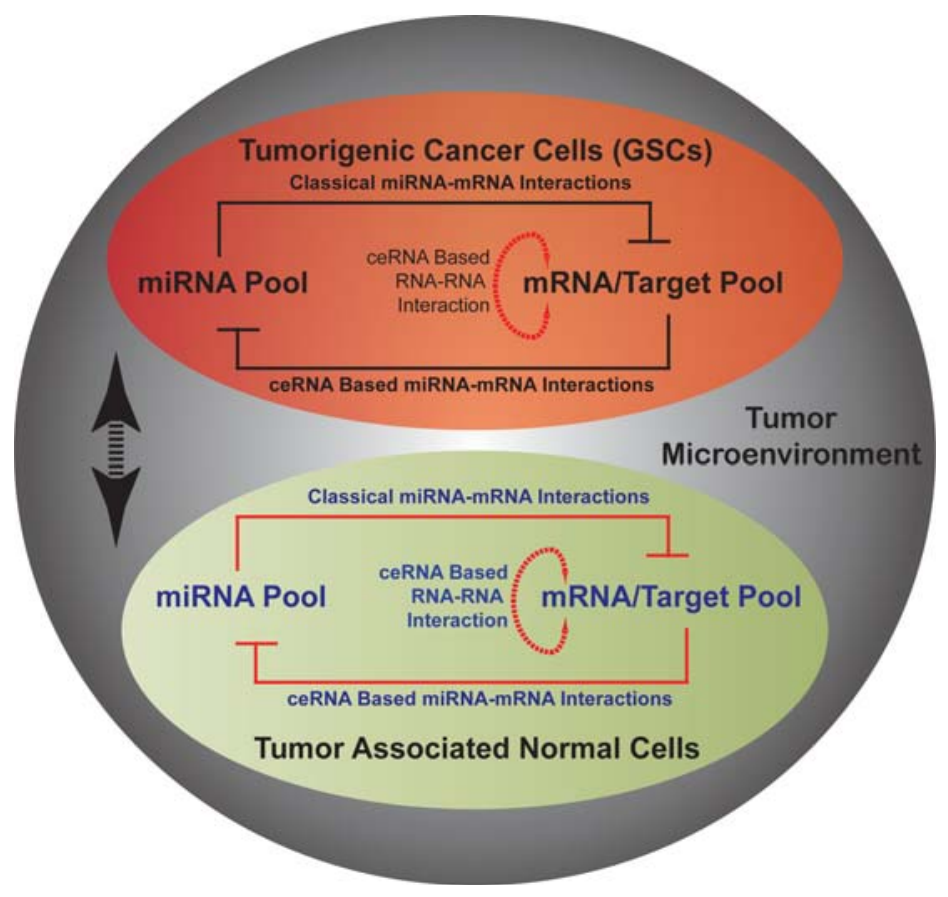




\section{Acknowledgements}

This review is dedicated to Abhijit Guha, whose mentorship and guidance is sorely missed. We would also like to thank Sameer Agnihotri for his comments on the manuscript.

\section{References}

1. Hanahan, D.; Weinberg, R.A. Hallmarks of cancer: The next generation. Cell 2011, 144, 646-674.

2. Stupp, R.; Hegi, M.E.; Mason, W.P.; van den Bent, M.J.; Taphoorn, M.J.; Janzer, R.C.; Ludwin, S.K.; Allgeier, A.; Fisher, B.; Belanger, K.; et al. Effects of radiotherapy with concomitant and adjuvant temozolomide versus radiotherapy alone on survival in glioblastoma in a randomised phase III study: 5-Year analysis of the EORTC-NCIC trial. Lancet Oncol. 2009, 10, 459-466.

3. Hjelmeland, A.B.; Lathia, J.D.; Sathornsumetee, S.; Rich, J.N. Twisted tango: Brain tumor neurovascular interactions. Nat. Neurosci. 2011, 14, 1375-1381.

4. Bao, S.; Wu, Q.; McLendon, R.E.; Hao, Y.; Shi, Q.; Hjelmeland, A.B.; Dewhirst, M.W.; Bigner, D.D.; Rich, J.N. Glioma stem cells promote radioresistance by preferential activation of the DNA damage response. Nature 2006, 444, 756-760.

5. Bao, S.; Wu, Q.; Sathornsumetee, S.; Hao, Y.; Li, Z.; Hjelmeland, A.B.; Shi, Q.; McLendon, R.E.; Bigner, D.D.; Rich, J.N. Stem cell-like glioma cells promote tumor angiogenesis through vascular endothelial growth factor. Cancer Res. 2006, 66, 7843-7848.

6. Calabrese, C.; Poppleton, H.; Kocak, M.; Hogg, T.L.; Fuller, C.; Hamner, B.; Oh, E.Y.; Gaber, M.W.; Finklestein, D.; Allen, M.; et al. A perivascular niche for brain tumor stem cells. Cancer Cell 2007, 11, 69-82.

7. Folkins, C.; Shaked, Y.; Man, S.; Tang, T.; Lee, C.R.; Zhu, Z.; Hoffman, R.M.; Kerbel, R.S. Glioma tumor stem-like cells promote tumor angiogenesis and vasculogenesis via vascular endothelial growth factor and stromal-derived factor 1. Cancer Res. 2009, 69, 7243-7251.

8. Gilbertson, R.J.; Rich, J.N. Making a tumour's bed: Glioblastoma stem cells and the vascular niche. Nat. Rev. Cancer 2007, 7, 733-736.

9. Heddleston, J.M.; Li, Z.; McLendon, R.E.; Hjelmeland, A.B.; Rich, J.N. The hypoxic microenvironment maintains glioblastoma stem cells and promotes reprogramming towards a cancer stem cell phenotype. Cell Cycle 2009, 8, 3274-3284.

10. Li, Z.; Bao, S.; Wu, Q.; Wang, H.; Eyler, C.; Sathornsumetee, S.; Shi, Q.; Cao, Y.; Lathia, J.; McLendon, R.E.; et al. Hypoxia-inducible factors regulate tumorigenic capacity of glioma stem cells. Cancer Cell 2009, 15, 501-513.

11. Liu, G.; Yuan, X.; Zeng, Z.; Tunici, P.; Ng, H.; Abdulkadir, I.R.; Lu, L.; Irvin, D.; Black, K.L.; $\mathrm{Yu}$, J.S. Analysis of gene expression and chemoresistance of cd133+ cancer stem cells in glioblastoma. Mol. Cancer 2006, 5, 67.

12. Salmaggi, A.; Boiardi, A.; Gelati, M.; Russo, A.; Calatozzolo, C.; Ciusani, E.; Sciacca, F.L.; Ottolina, A.; Parati, E.A.; La Porta, C.; et al. Glioblastoma-derived tumorospheres identify a population of tumor stem-like cells with angiogenic potential and enhanced multidrug resistance phenotype. Glia 2006, 54, 850-860. 
13. Singh, S.K.; Hawkins, C.; Clarke, I.D.; Squire, J.A.; Bayani, J.; Hide, T.; Henkelman, R.M.; Cusimano, M.D.; Dirks, P.B. Identification of human brain tumour initiating cells. Nature 2004, 432, 396-401.

14. Pistollato, F.; Abbadi, S.; Rampazzo, E.; Persano, L.; Della Puppa, A.; Frasson, C.; Sarto, E.; Scienza, R.; D’Avella, D.; Basso, G. Intratumoral hypoxic gradient drives stem cells distribution and mgmt expression in glioblastoma. Stem Cells 2010, 28, 851-862.

15. Baylin, S.B.; Jones, P.A. A decade of exploring the cancer epigenome-Biological and translational implications. Nat. Rev. Cancer 2011, 11, 726-734.

16. Nowell, P.C. The clonal evolution of tumor cell populations. Science 1976, 194, 23-28.

17. Bissell, M.J.; Hines, W.C. Why don't we get more cancer? A proposed role of the microenvironment in restraining cancer progression. Nat. Med. 2011, 17, 320-329.

18. Magee, J.A.; Piskounova, E.; Morrison, S.J. Cancer stem cells: Impact, heterogeneity, and uncertainty. Cancer Cell 2012, 21, 283-296.

19. Dick, J.E. Stem cell concepts renew cancer research. Blood 2008, 112, 4793-4807.

20. Reya, T.; Morrison, S.J.; Clarke, M.F.; Weissman, I.L. Stem cells, cancer, and cancer stem cells. Nature 2001, 414, 105-111.

21. Shackleton, M.; Quintana, E.; Fearon, E.R.; Morrison, S.J. Heterogeneity in cancer: Cancer stem cells versus clonal evolution. Cell 2009, 138, 822-829.

22. Eramo, A.; Ricci-Vitiani, L.; Zeuner, A.; Pallini, R.; Lotti, F.; Sette, G.; Pilozzi, E.; Larocca, L.M.; Peschle, C.; de Maria, R. Chemotherapy resistance of glioblastoma stem cells. Cell Death Differ. 2006, 13, 1238-1241.

23. Galli, R.; Binda, E.; Orfanelli, U.; Cipelletti, B.; Gritti, A.; de Vitis, S.; Fiocco, R.; Foroni, C.; Dimeco, F.; Vescovi, A. Isolation and characterization of tumorigenic, stem-like neural precursors from human glioblastoma. Cancer Res. 2004, 64, 7011-7021.

24. Sanai, N.; Alvarez-Buylla, A.; Berger, M.S. Neural stem cells and the origin of gliomas. N. Engl. J. Med. 2005, 353, 811-822.

25. Charles, N.; Ozawa, T.; Squatrito, M.; Bleau, A.M.; Brennan, C.W.; Hambardzumyan, D.; Holland, E.C. Perivascular nitric oxide activates notch signaling and promotes stem-like character in pdgf-induced glioma cells. Cell Stem Cell 2010, 6, 141-152.

26. Hambardzumyan, D.; Becher, O.J.; Rosenblum, M.K.; Pandolfi, P.P.; Manova-Todorova, K.; Holland, E.C. PI3K pathway regulates survival of cancer stem cells residing in the perivascular niche following radiation in medulloblastoma in vivo. Genes Dev. 2008, 22, 436-448.

27. Wu, A.; Wei, J.; Kong, L.Y.; Wang, Y.; Priebe, W.; Qiao, W.; Sawaya, R.; Heimberger, A.B. Glioma cancer stem cells induce immunosuppressive macrophages/microglia. Neurooncology 2010, 12, 1113-1125.

28. Deleyrolle, L.P.; Harding, A.; Cato, K.; Siebzehnrubl, F.A.; Rahman, M.; Azari, H.; Olson, S.; Gabrielli, B.; Osborne, G.; Vescovi, A.; et al. Evidence for label-retaining tumour-initiating cells in human glioblastoma. Brain 2011, 134, 1331-1343.

29. Knizetova, P.; Ehrmann, J.; Hlobilkova, A.; Vancova, I.; Kalita, O.; Kolar, Z.; Bartek, J. Autocrine regulation of glioblastoma cell cycle progression, viability and radioresistance through the vegf-vegfr2 (kdr) interplay. Cell Cycle 2008, 7, 2553-2561. 
30. Aboody, K.S.; Brown, A.; Rainov, N.G.; Bower, K.A.; Liu, S.; Yang, W.; Small, J.E.; Herrlinger, U.; Ourednik, V.; Black, P.M.; et al. Neural stem cells display extensive tropism for pathology in adult brain: Evidence from intracranial gliomas. Proc. Natl. Acad. Sci. USA 2000, 97, 12846-12851.

31. Assanah, M.C.; Bruce, J.N.; Suzuki, S.O.; Chen, A.; Goldman, J.E.; Canoll, P. PDGF stimulates the massive expansion of glial progenitors in the neonatal forebrain. Glia 2009, 57, 1835-1847.

32. Charles, N.A.; Holland, E.C.; Gilbertson, R.; Glass, R.; Kettenmann, H. The brain tumor microenvironment. Glia 2011, 59, 1169-1180.

33. Chirasani, S.R.; Sternjak, A.; Wend, P.; Momma, S.; Campos, B.; Herrmann, I.M.; Graf, D.; Mitsiadis, T.; Herold-Mende, C.; Besser, D.; et al. Bone morphogenetic protein-7 release from endogenous neural precursor cells suppresses the tumourigenicity of stem-like glioblastoma cells. Brain 2010, 133, 1961-1972.

34. Walzlein, J.H.; Synowitz, M.; Engels, B.; Markovic, D.S.; Gabrusiewicz, K.; Nikolaev, E.; Yoshikawa, K.; Kaminska, B.; Kempermann, G.; Uckert, W.; et al. The antitumorigenic response of neural precursors depends on subventricular proliferation and age. Stem Cells 2008, 26, 2945-2954.

35. Sanai, N.; Nguyen, T.; Ihrie, R.A.; Mirzadeh, Z.; Tsai, H.H.; Wong, M.; Gupta, N.; Berger, M.S.; Huang, E.; Garcia-Verdugo, J.M.; et al. Corridors of migrating neurons in the human brain and their decline during infancy. Nature 2011, 478, 382-386.

36. Dwain, I.; Xiangpeng, Y.; Zeng, Z.; Patricia, T.; Joh, S.Y. Neural stem cells-A promising potential therapy for brain tumors. Curr. Stem Cell Res. Ther. 2006, 1, 79-84.

37. Mokry, J.; Cizkova, D.; Filip, S.; Ehrmann, J.; Osterreicher, J.; Kolar, Z.; English, D. Nestin expression by newly formed human blood vessels. Stem Cells Dev. 2004, 13, 658-664.

38. Ricci-Vitiani, L.; Pallini, R.; Biffoni, M.; Todaro, M.; Invernici, G.; Cenci, T.; Maira, G.; Parati, E.A.; Stassi, G.; Larocca, L.M.; et al. Tumour vascularization via endothelial differentiation of glioblastoma stem-like cells. Nature 2010, 468, 824-828.

39. Soda, Y.; Marumoto, T.; Friedmann-Morvinski, D.; Soda, M.; Liu, F.; Michiue, H.; Pastorino, S.; Yang, M.; Hoffman, R.M.; Kesari, S.; et al. Transdifferentiation of glioblastoma cells into vascular endothelial cells. Proc. Natl. Acad. Sci. USA 2011, 108, 4274-4280.

40. Wang, R.; Chadalavada, K.; Wilshire, J.; Kowalik, U.; Hovinga, K.E.; Geber, A.; Fligelman, B.; Leversha, M.; Brennan, C.; Tabar, V. Glioblastoma stem-like cells give rise to tumour endothelium. Nature 2010, 468, 829-833.

41. Wen, P.Y.; Kesari, S. Malignant gliomas in adults. N. Engl. J. Med. 2008, 359, 492-507.

42. Brat, D.J.; van Meir, E.G. Glomeruloid microvascular proliferation orchestrated by VPF/VEGF: A new world of angiogenesis research. Am. J. Pathol. 2001, 158, 789-796.

43. Zadeh, G.; Koushan, K.; Baoping, Q.; Shannon, P.; Guha, A. Role of angiopoietin-2 in regulating growth and vascularity of astrocytomas. J. Oncol. 2010, 2010, 659231.

44. Wesseling, P.; Schlingemann, R.O.; Rietveld, F.J.; Link, M.; Burger, P.C.; Ruiter, D.J. Early and extensive contribution of pericytes/vascular smooth muscle cells to microvascular proliferation in glioblastoma multiforme: An immuno-light and immuno-electron microscopic study. J. Neuropathol. Exp. Neurol. 1995, 54, 304-310. 
45. Ahluwalia, M.S.; Gladson, C.L. Progress on antiangiogenic therapy for patients with malignant glioma. J. Oncol. 2010, 2010, 689018.

46. Bar, E.E.; Chaudhry, A.; Lin, A.; Fan, X.; Schreck, K.; Matsui, W.; Piccirillo, S.; Vescovi, A.L.; DiMeco, F.; Olivi, A.; et al. Cyclopamine-mediated hedgehog pathway inhibition depletes stem-like cancer cells in glioblastoma. Stem Cells 2007, 25, 2524-2533.

47. Becher, O.J.; Hambardzumyan, D.; Fomchenko, E.I.; Momota, H.; Mainwaring, L.; Bleau, A.M.; Katz, A.M.; Edgar, M.; Kenney, A.M.; Cordon-Cardo, C.; et al. Gli activity correlates with tumor grade in platelet-derived growth factor-induced gliomas. Cancer Res. 2008, 68, 2241-2249.

48. Clement, V.; Sanchez, P.; de Tribolet, N.; Radovanovic, I.; Ruiz i Altaba, A. HEDGEHOG-GLI1 signaling regulates human glioma growth, cancer stem cell self-renewal, and tumorigenicity. Curr. Biol. 2007, 17, 165-172.

49. Takezaki, T.; Hide, T.; Takanaga, H.; Nakamura, H.; Kuratsu, J.; Kondo, T. Essential role of the hedgehog signaling pathway in human glioma-initiating cells. Cancer Sci. 2011, 102, 1306-1312.

50. Dong, J.; Grunstein, J.; Tejada, M.; Peale, F.; Frantz, G.; Liang, W.C.; Bai, W.; Yu, L.; Kowalski, J.; Liang, X.; et al. VEGF-null cells require PDGFR alpha signaling-mediated stromal fibroblast recruitment for tumorigenesis. EMBO J. 2004, 23, 2800-2810.

51. Du, R.; Lu, K.V.; Petritsch, C.; Liu, P.; Ganss, R.; Passegue, E.; Song, H.; Vandenberg, S.; Johnson, R.S.; Werb, Z.; et al. HIF1alpha induces the recruitment of bone marrow-derived vascular modulatory cells to regulate tumor angiogenesis and invasion. Cancer Cell 2008, 13, 206-220.

52. Hashizume, H.; Baluk, P.; Morikawa, S.; McLean, J.W.; Thurston, G.; Roberge, S.; Jain, R.K.; McDonald, D.M. Openings between defective endothelial cells explain tumor vessel leakiness. Am. J. Pathol. 2000, 156, 1363-1380.

53. Morikawa, S.; Baluk, P.; Kaidoh, T.; Haskell, A.; Jain, R.K.; McDonald, D.M. Abnormalities in pericytes on blood vessels and endothelial sprouts in tumors. Am. J. Pathol. 2002, 160, 985-1000.

54. Warburg, O. On the origin of cancer cells. Science 1956, 123, 309-314.

55. Vander Heiden, M.G.; Cantley, L.C.; Thompson, C.B. Understanding the Warburg effect: The metabolic requirements of cell proliferation. Science 2009, 324, 1029-1033.

56. Cairns, R.A.; Harris, I.S.; Mak, T.W. Regulation of cancer cell metabolism. Nat. Rev. Cancer 2011, 11, 85-95.

57. Christofk, H.R.; Vander Heiden, M.G.; Harris, M.H.; Ramanathan, A.; Gerszten, R.E.; Wei, R.; Fleming, M.D.; Schreiber, S.L.; Cantley, L.C. The M2 splice isoform of pyruvate kinase is important for cancer metabolism and tumour growth. Nature 2008, 452, 230-233.

58. Christofk, H.R.; Vander Heiden, M.G.; Wu, N.; Asara, J.M.; Cantley, L.C. Pyruvate kinase M2 is a phosphotyrosine-binding protein. Nature 2008, 452, 181-186.

59. Wolf, A.; Agnihotri, S.; Micallef, J.; Mukherjee, J.; Sabha, N.; Cairns, R.; Hawkins, C.; Guha, A. Hexokinase 2 is a key mediator of aerobic glycolysis and promotes tumor growth in human glioblastoma multiforme. J. Exp. Med. 2011, 208, 313-326.

60. Shim, H.; Dolde, C.; Lewis, B.C.; Wu, C.S.; Dang, G.; Jungmann, R.A.; Dalla-Favera, R.; Dang, C.V. c-Myc transactivation of ldh-a: Implications for tumor metabolism and growth. Proc. Natl. Acad. Sci. USA 1997, 94, 6658-6663. 
61. Fantin, V.R.; St-Pierre, J.; Leder, P. Attenuation of LDH-A expression uncovers a link between glycolysis, mitochondrial physiology, and tumor maintenance. Cancer Cell 2006, 9, 425-434.

62. Michelakis, E.D.; Sutendra, G.; Dromparis, P.; Webster, L.; Haromy, A.; Niven, E.; Maguire, C.; Gammer, T.L.; Mackey, J.R.; Fulton, D.; et al. Metabolic modulation of glioblastoma with dichloroacetate. Sci. Transl. Med. 2010, 2, 31 ra34.

63. Snuderl, M.; Fazlollahi, L.; Le, L.P.; Nitta, M.; Zhelyazkova, B.H.; Davidson, C.J.; Akhavanfard, S.; Cahill, D.P.; Aldape, K.D.; Betensky, R.A.; et al. Mosaic amplification of multiple receptor tyrosine kinase genes in glioblastoma. Cancer Cell 2011, 20, 810-817.

64. Bristow, R.G.; Hill, R.P. Hypoxia and metabolism. Hypoxia, DNA repair and genetic instability. Nat. Rev. Cancer 2008, 8, 180-192.

65. Barker, F.G., 2nd; Davis, R.L.; Chang, S.M.; Prados, M.D. Necrosis as a prognostic factor in glioblastoma multiforme. Cancer 1996, 77, 1161-1166.

66. Brat, D.J.; Kaur, B.; van Meir, E.G. Genetic modulation of hypoxia induced gene expression and angiogenesis: Relevance to brain tumors. Front. Biosci. 2003, 8, d100-d116.

67. Burger, P.C.; Vollmer, R.T. Histologic factors of prognostic significance in the glioblastoma multiforme. Cancer 1980, 46, 1179-1186.

68. Evans, S.M.; Judy, K.D.; Dunphy, I.; Jenkins, W.T.; Nelson, P.T.; Collins, R.; Wileyto, E.P.; Jenkins, K.; Hahn, S.M.; Stevens, C.W.; et al. Comparative measurements of hypoxia in human brain tumors using needle electrodes and EF5 binding. Cancer Res. 2004, 64, 1886-1892.

69. Wang, G.L.; Jiang, B.H.; Rue, E.A.; Semenza, G.L. Hypoxia-inducible factor 1 is a basic-helixloop-helix-PAS heterodimer regulated by cellular O2 tension. Proc. Natl. Acad. Sci. USA 1995, 92, 5510-5514.

70. Kim, J.W.; Gao, P.; Dang, C.V. Effects of hypoxia on tumor metabolism. Cancer Metastasis Rev. 2007, 26, 291-298.

71. Semenza, G.L. HIF-1: Upstream and downstream of cancer metabolism. Curr. Opin. Genet. Dev. 2010, 20, 51-56.

72. Denko, N.C. Hypoxia, HIF1 and glucose metabolism in the solid tumour. Nat. Rev. Cancer 2008, 8, 705-713.

73. Carro, M.S.; Lim, W.K.; Alvarez, M.J.; Bollo, R.J.; Zhao, X.; Snyder, E.Y.; Sulman, E.P.; Anne, S.L.; Doetsch, F.; Colman, H.; et al. The transcriptional network for mesenchymal transformation of brain tumours. Nature 2010, 463, 318-325.

74. Maslov, S.; Sneppen, K. Specificity and stability in topology of protein networks. Science 2002, 296, 910-913.

75. Kim, J.; Chu, J.; Shen, X.; Wang, J.; Orkin, S.H. An extended transcriptional network for pluripotency of embryonic stem cells. Cell 2008, 132, 1049-1061.

76. Orkin, S.H.; Wang, J.; Kim, J.; Chu, J.; Rao, S.; Theunissen, T.W.; Shen, X.; Levasseur, D.N. The transcriptional network controlling pluripotency in ES cells. Cold Spring Harb. Symp. Quant. Biol. 2008, 73, 195-202.

77. Sumazin, P.; Yang, X.; Chiu, H.S.; Chung, W.J.; Iyer, A.; Llobet-Navas, D.; Rajbhandari, P.; Bansal, M.; Guarnieri, P.; Silva, J.; et al. An extensive microrna-mediated network of rna-rna interactions regulates established oncogenic pathways in glioblastoma. Cell 2011, 147, 370-381. 
78. Xiao, F.; Zuo, Z.; Cai, G.; Kang, S.; Gao, X.; Li, T. Mirecords: An integrated resource for microrna-target interactions. Nucleic Acids Res. 2009, 37, D105-D110.

79. Cai, X.; Hagedorn, C.H.; Cullen, B.R. Human micrornas are processed from capped, polyadenylated transcripts that can also function as mrnas. RNA 2004, 10, 1957-1966.

80. Lee, Y.; Kim, M.; Han, J.; Yeom, K.H.; Lee, S.; Baek, S.H.; Kim, V.N. MicroRNA genes are transcribed by rna polymerase II. EMBO J. 2004, 23, 4051-4060.

81. Seitz, H.; Youngson, N.; Lin, S.P.; Dalbert, S.; Paulsen, M.; Bachellerie, J.P.; Ferguson-Smith, A.C.; Cavaille, J. Imprinted microrna genes transcribed antisense to a reciprocally imprinted retrotransposon-like gene. Nat. Genet. 2003, 34, 261-262.

82. Lee, Y.; Jeon, K.; Lee, J.T.; Kim, S.; Kim, V.N. MicroRNA maturation: Stepwise processing and subcellular localization. EMBO J. 2002, 21, 4663-4670.

83. Okamura, K.; Hagen, J.W.; Duan, H.; Tyler, D.M.; Lai, E.C. The mirtron pathway generates microrna-class regulatory RNAs in drosophila. Cell 2007, 130, 89-100.

84. Ruby, J.G.; Jan, C.H.; Bartel, D.P. Intronic microRNA precursors that bypass Drosha processing. Nature 2007, 448, 83-86.

85. Bohnsack, M.T.; Czaplinski, K.; Gorlich, D. Exportin 5 is a RanGTP-dependent dsRNA-binding protein that mediates nuclear export of pre-miRNAs. RNA 2004, 10, 185-191.

86. Lund, E.; Guttinger, S.; Calado, A.; Dahlberg, J.E.; Kutay, U. Nuclear export of microRNA precursors. Science 2004, 303, 95-98.

87. Yi, R.; Qin, Y.; Macara, I.G.; Cullen, B.R. Exportin-5 mediates the nuclear export of pre-microRNAs and short hairpin RNAs. Genes Dev. 2003, 17, 3011-3016.

88. Zhang, H.; Kolb, F.A.; Jaskiewicz, L.; Westhof, E.; Filipowicz, W. Single processing center models for human Dicer and bacterial RNase III. Cell 2004, 118, 57-68.

89. Mourelatos, Z.; Dostie, J.; Paushkin, S.; Sharma, A.; Charroux, B.; Abel, L.; Rappsilber, J.; Mann, M.; Dreyfuss, G. Mirnps: A novel class of ribonucleoproteins containing numerous microRNAs. Genes Dev. 2002, 16, 720-728.

90. Liu, J.; Carmell, M.A.; Rivas, F.V.; Marsden, C.G.; Thomson, J.M.; Song, J.J.; Hammond, S.M.; Joshua-Tor, L.; Hannon, G.J. Argonaute2 is the catalytic engine of mammalian RNAi. Science 2004, 305, 1437-1441.

91. Meister, G.; Landthaler, M.; Patkaniowska, A.; Dorsett, Y.; Teng, G.; Tuschl, T. Human argonaute2 mediates RNA cleavage targeted by miRNAs and siRNAs. Mol. Cell 2004, 15, 185-197.

92. Pillai, R.S.; Artus, C.G.; Filipowicz, W. Tethering of human ago proteins to mRNA mimics the miRNA-mediated repression of protein synthesis. RNA 2004, 10, 1518-1525.

93. Brennecke, J.; Stark, A.; Russell, R.B.; Cohen, S.M. Principles of microRNA-target recognition. PLoS Biol. 2005, 3, e85.

94. Doench, J.G.; Sharp, P.A. Specificity of microRNA target selection in translational repression. Genes Dev. 2004, 18, 504-511.

95. Grimson, A.; Farh, K.K.; Johnston, W.K.; Garrett-Engele, P.; Lim, L.P.; Bartel, D.P. MicroRNA targeting specificity in mammals: Determinants beyond seed pairing. Mol. Cell 2007, 27, 91-105.

96. Lewis, B.P.; Burge, C.B.; Bartel, D.P. Conserved seed pairing, often flanked by adenosines, indicates that thousands of human genes are microRNA targets. Cell 2005, 120, 15-20. 
97. Nielsen, C.B.; Shomron, N.; Sandberg, R.; Hornstein, E.; Kitzman, J.; Burge, C.B. Determinants of targeting by endogenous and exogenous microRNAs and siRNAs. RNA 2007, 13, 1894-1910.

98. Rana, T.M. Illuminating the silence: Understanding the structure and function of small RNAs. Nat. Rev. Mol. Cell Biol. 2007, 8, 23-36.

99. Zhang, Y.; Dutta, A.; Abounader, R. The role of microRNAs in glioma initiation and progression. Front. Biosci. 2012, 17, 700-712.

100. Sana, J.; Hajduch, M.; Michalek, J.; Vyzula, R.; Slaby, O. MicroRNAs and glioblastoma: Roles in core signalling pathways and potential clinical implications. J. Cell. Mol. Med. 2011, 15, 1636-1644.

101. Chen, L.; Zhang, J.; Han, L.; Zhang, A.; Zhang, C.; Zheng, Y.; Jiang, T.; Pu, P.; Jiang, C.; Kang, C. Downregulation of miR-221/222 sensitizes glioma cells to temozolomide by regulating apoptosis independently of p53 status. Oncol. Rep. 2012, 27, 854-860.

102. Quintavalle, C.; Garofalo, M.; Zanca, C.; Romano, G.; Iaboni, M.; del Basso de Caro, M.; Martinez-Montero, J.C.; Incoronato, M.; Nuovo, G.; Croce, C.M.; et al. MiR-221/222 overexpession in human glioblastoma increases invasiveness by targeting the protein phosphate ptpmu. Oncogene 2012, 31, 858-868.

103. Hsu, S.D.; Lin, F.M.; Wu, W.Y.; Liang, C.; Huang, W.C.; Chan, W.L.; Tsai, W.T.; Chen, G.Z.; Lee, C.J.; Chiu, C.M.; et al. Mirtarbase: A database curates experimentally validated microRNA-target interactions. Nucleic Acids Res. 2011, 39, D163-D169.

104. Huang da, W.; Sherman, B.T.; Lempicki, R.A. Bioinformatics enrichment tools: Paths toward the comprehensive functional analysis of large gene lists. Nucleic Acids Res. 2009, 37, 1-13.

105. Dong, H.; Luo, L.; Hong, S.; Siu, H.; Xiao, Y.; Jin, L.; Chen, R.; Xiong, M. Integrated analysis of mutations, miRNA and mRNA expression in glioblastoma. BMC Syst. Biol. 2010, 4, 163.

106. Gal, H.; Pandi, G.; Kanner, A.A.; Ram, Z.; Lithwick-Yanai, G.; Amariglio, N.; Rechavi, G.; Givol, D. MiR-451 and imatinib mesylate inhibit tumor growth of glioblastoma stem cells. Biochem. Biophys. Res. Commun. 2008, 376, 86-90.

107. Godlewski, J.; Newton, H.B.; Chiocca, E.A.; Lawler, S.E. MicroRNAs and glioblastoma; the stem cell connection. Cell Death Differ. 2010, 17, 221-228.

108. Kefas, B.; Godlewski, J.; Comeau, L.; Li, Y.; Abounader, R.; Hawkinson, M.; Lee, J.; Fine, H.; Chiocca, E.A.; Lawler, S.; et al. MicroRNA-7 inhibits the epidermal growth factor receptor and the Akt pathway and is down-regulated in glioblastoma. Cancer Res. 2008, 68, 3566-3572.

109. Silber, J.; Lim, D.A.; Petritsch, C.; Persson, A.I.; Maunakea, A.K.; Yu, M.; Vandenberg, S.R.; Ginzinger, D.G.; James, C.D.; Costello, J.F.; et al. MiR-124 and miR-137 inhibit proliferation of glioblastoma multiforme cells and induce differentiation of brain tumor stem cells. BMC Med. 2008, 6, 14.

110. Zhang, Y.; Chao, T.; Li, R.; Liu, W.; Chen, Y.; Yan, X.; Gong, Y.; Yin, B.; Qiang, B.; Zhao, J.; et al. MicroRNA-128 inhibits glioma cells proliferation by targeting transcription factor E2F3a. J. Mol. Med. 2009, 87, 43-51.

111. Fan, X.; Khaki, L.; Zhu, T.S.; Soules, M.E.; Talsma, C.E.; Gul, N.; Koh, C.; Zhang, J.; Li, Y.M.; Maciaczyk, J.; et al. Notch pathway blockade depletes CD133-positive glioblastoma cells and inhibits growth of tumor neurospheres and xenografts. Stem Cells 2010, 28, 5-16. 
112. Li, Y.; Guessous, F.; Zhang, Y.; Dipierro, C.; Kefas, B.; Johnson, E.; Marcinkiewicz, L.; Jiang, J.; Yang, Y.; Schmittgen, T.D.; et al. MicroRNA-34a inhibits glioblastoma growth by targeting multiple oncogenes. Cancer Res. 2009, 69, 7569-7576.

113. Kefas, B.; Comeau, L.; Floyd, D.H.; Seleverstov, O.; Godlewski, J.; Schmittgen, T.; Jiang, J.; diPierro, C.G.; Li, Y.; Chiocca, E.A.; et al. The neuronal microRNA miR-326 acts in a feedback loop with notch and has therapeutic potential against brain tumors. J. Neurosci. 2009, 29, 15161-15168.

114. Chen, Y.; Gorski, D.H. Regulation of angiogenesis through a microRNA (miR-130a) that downregulates antiangiogenic homeobox genes gax and hoxa5. Blood 2008, 111, 1217-1226.

115. Dews, M.; Homayouni, A.; Yu, D.; Murphy, D.; Sevignani, C.; Wentzel, E.; Furth, E.E.; Lee, W.M.; Enders, G.H.; Mendell, J.T.; et al. Augmentation of tumor angiogenesis by a Myc-activated microRNA cluster. Nat. Genet. 2006, 38, 1060-1065.

116. Fasanaro, P.; D’Alessandra, Y.; di Stefano, V.; Melchionna, R.; Romani, S.; Pompilio, G.; Capogrossi, M.C.; Martelli, F. MicroRNA-210 modulates endothelial cell response to hypoxia and inhibits the receptor tyrosine kinase ligand Ephrin-A3. J. Biol. Chem. 2008, 283, 15878-15883.

117. Fish, J.E.; Santoro, M.M.; Morton, S.U.; Yu, S.; Yeh, R.F.; Wythe, J.D.; Ivey, K.N.; Bruneau, B.G.; Stainier, D.Y.; Srivastava, D. miR-126 Regulates angiogenic signaling and vascular integrity. Dev. Cell 2008, 15, 272-284.

118. Hua, Z.; Lv, Q.; Ye, W.; Wong, C.K.; Cai, G.; Gu, D.; Ji, Y.; Zhao, C.; Wang, J.; Yang, B.B.; et al. MiRNA-directed regulation of VEGF and other angiogenic factors under hypoxia. PLoS One 2006, 1, e116.

119. Kuehbacher, A.; Urbich, C.; Zeiher, A.M.; Dimmeler, S. Role of dicer and drosha for endothelial microRNA expression and angiogenesis. Circ. Res. 2007, 101, 59-68.

120. Kuhnert, F.; Mancuso, M.R.; Hampton, J.; Stankunas, K.; Asano, T.; Chen, C.Z.; Kuo, C.J. Attribution of vascular phenotypes of the murine EGFL7 locus to the microRNA miR-126. Development 2008, 135, 3989-3993.

121. Lee, D.Y.; Deng, Z.; Wang, C.H.; Yang, B.B. MicroRNA-378 promotes cell survival, tumor growth, and angiogenesis by targeting SuFu and Fus-1 expression. Proc. Natl. Acad. Sci. USA 2007, 104, 20350-20355.

122. Pulkkinen, K.; Malm, T.; Turunen, M.; Koistinaho, J.; Yla-Herttuala, S. Hypoxia induces microRNA miR-210 in vitro and in vivo Ephrin-a3 and neuronal pentraxin 1 are potentially regulated by miR-210. FEBS Lett. 2008, 582, 2397-2401.

123. Wang, S.; Aurora, A.B.; Johnson, B.A.; Qi, X.; McAnally, J.; Hill, J.A.; Richardson, J.A.; Bassel-Duby, R.; Olson, E.N. The endothelial-specific microRNA miR-126 governs vascular integrity and angiogenesis. Dev. Cell 2008, 15, 261-271.

124. Wurdinger, T.; Tannous, B.A.; Saydam, O.; Skog, J.; Grau, S.; Soutschek, J.; Weissleder, R.; Breakefield, X.O.; Krichevsky, A.M. miR-296 Regulates growth factor receptor overexpression in angiogenic endothelial cells. Cancer Cell 2008, 14, 382-393.

125. Smits, M.; Wurdinger, T.; van Het Hof, B.; Drexhage, J.A.; Geerts, D.; Wesseling, P.; Noske, D.P.; Vandertop, W.P.; de Vries, H.E.; Reijerkerk, A. Myc-associated zinc finger protein (MAZ) is regulated by miR-125b and mediates VEGF-induced angiogenesis in glioblastoma. FASEB J. 2012, doi:10.1096/fj.11-202820. 
126. Smits, M.; Mir, S.E.; Nilsson, R.J.; van der Stoop, P.M.; Niers, J.M.; Marquez, V.E.; Cloos, J.; Breakefield, X.O.; Krichevsky, A.M.; Noske, D.P.; et al. Down-regulation of miR-101 in endothelial cells promotes blood vessel formation through reduced repression of EZH2. PLoS One 2011, 6, e16282.

127. Poliseno, L.; Tuccoli, A.; Mariani, L.; Evangelista, M.; Citti, L.; Woods, K.; Mercatanti, A.; Hammond, S.; Rainaldi, G. MicroRNAs modulate the angiogenic properties of huvecs. Blood 2006, 108, 3068-3071.

128. Wang, X.H.; Qian, R.Z.; Zhang, W.; Chen, S.F.; Jin, H.M.; Hu, R.M. MicroRNA-320 expression in myocardial microvascular endothelial cells and its relationship with insulin-like growth factor-1 in type 2 diabetic rats. Clin. Exp. Pharmacol. Physiol. 2009, 36, 181-188.

129. Godlewski, J.; Nowicki, M.O.; Bronisz, A.; Nuovo, G.; Palatini, J.; de Lay, M.; van Brocklyn, J.; Ostrowski, M.C.; Chiocca, E.A.; Lawler, S.E. MicroRNA-451 regulates LKB1/AMPK signaling and allows adaptation to metabolic stress in glioma cells. Mol. Cell 2010, 37, 620-632.

130. Gao, P.; Tchernyshyov, I.; Chang, T.C.; Lee, Y.S.; Kita, K.; Ochi, T.; Zeller, K.I.; de Marzo, A.M.; van Eyk, J.E.; Mendell, J.T.; et al. c-Myc suppression of miR-23a/b enhances mitochondrial glutaminase expression and glutamine metabolism. Nature 2009, 458, 762-765.

131. Olive, V.; Bennett, M.J.; Walker, J.C.; Ma, C.; Jiang, I.; Cordon-Cardo, C.; Li, Q.J.; Lowe, S.W.; Hannon, G.J.; He, L. miR-19 Is a key oncogenic component of miR-17-92. Genes Dev. 2009, 23, 2839-2849.

132. Kulshreshtha, R.; Ferracin, M.; Negrini, M.; Calin, G.A.; Davuluri, R.V.; Ivan, M. Regulation of microRNA expression: The hypoxic component. Cell Cycle 2007, 6, 1426-1431.

133. Chan, S.Y.; Zhang, Y.Y.; Hemann, C.; Mahoney, C.E.; Zweier, J.L.; Loscalzo, J. MicroRNA-210 controls mitochondrial metabolism during hypoxia by repressing the iron-sulfur cluster assembly proteins ISCU1/2. Cell Metab. 2009, 10, 273-284.

134. Cascio, S.; D’Andrea, A.; Ferla, R.; Surmacz, E.; Gulotta, E.; Amodeo, V.; Bazan, V.; Gebbia, N.; Russo, A. miR-20b Modulates VEGF expression by targeting HIF-1 $\alpha$ and STAT3 in MCF-7 breast cancer cells. J. Cell. Physiol. 2010, 224, 242-249.

135. Rane, S.; He, M.; Sayed, D.; Vashistha, H.; Malhotra, A.; Sadoshima, J.; Vatner, D.E.; Vatner, S.F.; Abdellatif, M. Downregulation of miR-199a derepresses hypoxia-inducible factor-1 $\alpha$ and Sirtuin 1 and recapitulates hypoxia preconditioning in cardiac myocytes. Circ. Res. 2009, 104, 879-886.

136. Stiles, C.D.; Rowitch, D.H. Glioma stem cells: A midterm exam. Neuron 2008, 58, 832-846.

137. Shi, L.; Zhang, J.; Pan, T.; Zhou, J.; Gong, W.; Liu, N.; Fu, Z.; You, Y. MiR-125b is critical for the suppression of human U251 glioma stem cell proliferation. Brain Res. 2010, 1312, 120-126.

138. Bruggeman, S.W.; Hulsman, D.; Tanger, E.; Buckle, T.; Blom, M.; Zevenhoven, J.; van Tellingen, O.; van Lohuizen, M. Bmil controls tumor development in an Ink4a/Arf-independent manner in a mouse model for glioma. Cancer Cell 2007, 12, 328-341.

139. Dirks, P. Bmil and cell of origin determinants of brain tumor phenotype. Cancer Cell 2007, 12, 295-297.

140. Jin, X.; Yin, J.; Kim, S.H.; Sohn, Y.W.; Beck, S.; Lim, Y.C.; Nam, D.H.; Choi, Y.J.; Kim, H. EGFR-AKT-SMAD signaling promotes formation of glioma stem-like cells and tumor angiogenesis by ID3-driven cytokine induction. Cancer Res. 2011, 71, 7125-7134. 
141. Piccirillo, S.G.; Reynolds, B.A.; Zanetti, N.; Lamorte, G.; Binda, E.; Broggi, G.; Brem, H.; Olivi, A.; Dimeco, F.; Vescovi, A.L. Bone morphogenetic proteins inhibit the tumorigenic potential of human brain tumour-initiating cells. Nature 2006, 444, 761-765.

142. Shih, A.H.; Holland, E.C. Notch signaling enhances nestin expression in gliomas. Neoplasia 2006, 8, 1072-1082.

143. Wang, J.; Wakeman, T.P.; Lathia, J.D.; Hjelmeland, A.B.; Wang, X.F.; White, R.R.; Rich, J.N.; Sullenger, B.A. Notch promotes radioresistance of glioma stem cells. Stem Cells 2010, 28, 17-28.

144. Zhang, X.P.; Zheng, G.; Zou, L.; Liu, H.L.; Hou, L.H.; Zhou, P.; Yin, D.D.; Zheng, Q.J.; Liang, L.; Zhang, S.Z; et al. Notch activation promotes cell proliferation and the formation of neural stem cell-like colonies in human glioma cells. Mol. Cell. Biochem. 2008, 307, 101-108.

145. Cao, Y.; Nagesh, V.; Hamstra, D.; Tsien, C.I.; Ross, B.D.; Chenevert, T.L.; Junck, L.; Lawrence, T.S. The extent and severity of vascular leakage as evidence of tumor aggressiveness in high-grade gliomas. Cancer Res. 2006, 66, 8912-8917.

146. Rutten, E.H.; Doesburg, W.H.; Slooff, J.L. Histologic factors in the grading and prognosis of astrocytoma grade I-IV. J. Neurooncol. 1992, 13, 223-230.

147. Wang, S.; Olson, E.N. Angiomirs-Key regulators of angiogenesis. Curr. Opin. Genet. Dev. 2009, 19, 205-211.

148. Kefas, B.; Comeau, L.; Erdle, N.; Montgomery, E.; Amos, S.; Purow, B. Pyruvate kinase M2 is a target of the tumor-suppressive microRNA-326 and regulates the survival of glioma cells. Neurooncology 2010, 12, 1102-1112.

149. Chan, J.A.; Krichevsky, A.M.; Kosik, K.S. MicroRNA-21 is an antiapoptotic factor in human glioblastoma cells. Cancer Res. 2005, 65, 6029-6033.

150. Godlewski, J.; Nowicki, M.O.; Bronisz, A.; Williams, S.; Otsuki, A.; Nuovo, G.; Raychaudhury, A.; Newton, H.B.; Chiocca, E.A.; Lawler, S. Targeting of the Bmi-1 oncogene/stem cell renewal factor by microRNA-128 inhibits glioma proliferation and self-renewal. Cancer Res. 2008, 68, 9125-9130.

151. Huse, J.T.; Brennan, C.; Hambardzumyan, D.; Wee, B.; Pena, J.; Rouhanifard, S.H.; Sohn-Lee, C.; le Sage, C.; Agami, R.; Tuschl, T.; et al. The PTEN-regulating microRNA miR-26a is amplified in high-grade glioma and facilitates gliomagenesis in vivo. Genes Dev. 2009, 23, 1327-1337.

152. Nan, Y.; Han, L.; Zhang, A.; Wang, G.; Jia, Z.; Yang, Y.; Yue, X.; Pu, P.; Zhong, Y.; Kang, C. MiRNA-451 plays a role as tumor suppressor in human glioma cells. Brain Res. 2010, 1359, 14-21.

153. Wang, Y.; Wang, X.; Zhang, J.; Sun, G.; Luo, H.; Kang, C.; Pu, P.; Jiang, T.; Liu, N.; You, Y. MicroRNAs involved in the EGFR/PTEN/AKT pathway in gliomas. J. Neurooncol. 2012, 106, 217-224.

154. Zhang, J.; Han, L.; Ge, Y.; Zhou, X.; Zhang, A.; Zhang, C.; Zhong, Y.; You, Y.; Pu, P.; Kang, C. miR-221/222 Promote malignant progression of glioma through activation of the Akt pathway. Int. J. Oncol. 2010, 36, 913-920.

155. Zhou, X.; Ren, Y.; Moore, L.; Mei, M.; You, Y.; Xu, P.; Wang, B.; Wang, G.; Jia, Z.; Pu, P.; et al. Downregulation of miR-21 inhibits EGFR pathway and suppresses the growth of human glioblastoma cells independent of pten status. Lab. Invest. 2010, 90, 144-155.

156. Papagiannakopoulos, T.; Shapiro, A.; Kosik, K.S. MicroRNA-21 targets a network of key tumor-suppressive pathways in glioblastoma cells. Cancer Res. 2008, 68, 8164-8172. 
157. Suh, S.S.; Yoo, J.Y.; Nuovo, G.J.; Jeon, Y.J.; Kim, S.; Lee, T.J.; Kim, T.; Bakacs, A.; Alder, H.; Kaur, B.; et al. MicroRNAs/TP53 feedback circuitry in glioblastoma multiforme. Proc. Natl. Acad. Sci. USA 2012, 109, 5316-5321.

158. Luan, S.; Sun, L.; Huang, F. MicroRNA-34a: A novel tumor suppressor in p53-mutant glioma cell line U251. Arch. Med. Res. 2010, 41, 67-74.

159. Jiang, S.; Zhang, L.F.; Zhang, H.W.; Hu, S.; Lu, M.H.; Liang, S.; Li, B.; Li, Y.; Li, D.; Wang, E.D.; et al. A novel miR-155/miR-143 cascade controls glycolysis by regulating hexokinase 2 in breast cancer cells. EMBO J. 2012, 31, 1985-1998.

160. Wolf, A.; Agnihotri, S.; Munoz, D.; Guha, A. Developmental profile and regulation of the glycolytic enzyme hexokinase 2 in normal brain and glioblastoma multiforme. Neurobiol. Dis. 2011, 44, 84-91.

161. O’Donnell, K.A.; Wentzel, E.A.; Zeller, K.I.; Dang, C.V.; Mendell, J.T. c-Myc-regulated microRNAs modulate E2F1 expression. Nature 2005, 435, 839-843.

162. Chan, S.Y.; Loscalzo, J. MicroRNA-210: A unique and pleiotropic hypoxamir. Cell Cycle 2010, 9, 1072-1083.

163. Lages, E.; Guttin, A.; El Atifi, M.; Ramus, C.; Ipas, H.; Dupre, I.; Rolland, D.; Salon, C.; Godfraind, C.; deFraipont, F.; et al. MicroRNA and target protein patterns reveal physiopathological features of glioma subtypes. PLoS One 2011, 6, e20600.

164. Poliseno, L.; Salmena, L.; Zhang, J.; Carver, B.; Haveman, W.J.; Pandolfi, P.P. A coding-independent function of gene and pseudogene mRNAs regulates tumour biology. Nature 2010, 465, 1033-1038.

165. Salmena, L.; Poliseno, L.; Tay, Y.; Kats, L.; Pandolfi, P.P. A ceRNA hypothesis: The Rosetta Stone of a hidden RNA language? Cell 2011, 146, 353-358.

166. Sumazin, P.; Yang, X.; Chiu, H.S.; Chung, W.J.; Iyer, A.; Llobet-Navas, D.; Rajbhandari, P.; Bansal, M.; Guarnieri, P.; Silva, J.; et al. An extensive microRNA-mediated network of RNA-RNA interactions regulates established oncogenic pathways in glioblastoma. Cell 2011, 147, 370-381.

167. Tay, Y.; Kats, L.; Salmena, L.; Weiss, D.; Tan, S.M.; Ala, U.; Karreth, F.; Poliseno, L.; Provero, P.; di Cunto, F.; et al. Coding-independent regulation of the tumor suppressor pten by competing endogenous mRNAs. Cell 2011, 147, 344-357.

(C) 2012 by the authors; licensee MDPI, Basel, Switzerland. This article is an open access article distributed under the terms and conditions of the Creative Commons Attribution license (http://creativecommons.org/licenses/by/3.0/). 